\title{
INTERNAL ROTATION OF THE RED-GIANT STAR KIC 4448777 BY MEANS OF ASTEROSEISMIC INVERSION
}

\author{
M. P. Di Mauro ${ }^{1}$, R. Ventura ${ }^{2}$, D. Cardini ${ }^{1}$, D. Stello ${ }^{3,4}$, J. Christensen-Dalsgaard ${ }^{4}$, W. A. Dziembowski ${ }^{5,6}$,

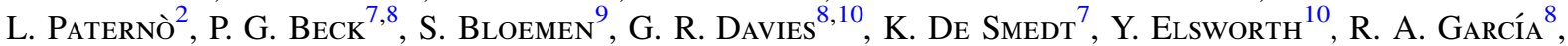 \\ S. HeKKER ${ }^{4,11,12}$, B. MOSSER ${ }^{13}$, AND A. TKACHENKo ${ }^{7}$ \\ ${ }^{1}$ INAF, IAPS Istituto di Astrofisica e Planetologia Spaziali, Roma, Italy \\ ${ }^{2}$ INAF, Astrophysical Observatory of Catania, Catania, Italy \\ ${ }^{3}$ Sydney Institute for Astronomy, School of Physics, University of Sydney, Australia \\ ${ }^{4}$ Stellar Astrophysics Centre, Department of Physics and Astronomy, Aarhus University, Ny Munkegade 120, DK-8000 Aarhus C, Denmark \\ ${ }^{5}$ Warsaw University Observatory, Al. Ujazdowskie 4, 00-478 Warsaw, Poland \\ ${ }^{6}$ Copernicus Astronomical Center, ul. Bartycka 18, 00-716 Warsaw, Poland \\ ${ }^{7}$ Instituut voor Sterrenkunde, Katholieke Universiteit Leuven, Belgium \\ ${ }^{8}$ Laboratoire AIM, CEA/DSM-CNRS-Univ. Paris Diderot, IRFU/Sap, Centre de Saclay, F-91191 Gif-sur-Yvette Cedex, France \\ ${ }^{9}$ Department of Astrophysics, IMAPP, Radboud University Nijmegen, P.O. Box 9010, NL-6500 GL, Nijmegen, The Netherlands \\ ${ }^{10}$ School of Physics and Astronomy, University of Birmingham, UK \\ ${ }^{11}$ Astronomical Institute Anton Pannekoek, University of Amsterdam, The Netherlands \\ ${ }^{12}$ Max Planck Institute for Solar System Research, Justus-von-Liebig-Weg 3, D-37077 Göttingen, Germany \\ ${ }^{13}$ LESIA, PSL Research University, CNRS, Universitè Pierre et Marie Curie, Université Denis Diderot, Observatoire de Paris, Meudon Cedex, France \\ Received 2015 March 17; accepted 2015 November 18; published 2016 January 22
}

\begin{abstract}
We study the dynamics of the stellar interior of the early red-giant star KIC 4448777 by asteroseismic inversion of 14 splittings of the dipole mixed modes obtained from Kepler observations. In order to overcome the complexity of the oscillation pattern typical of red-giant stars, we present a procedure to extract the rotational splittings from the power spectrum. We find not only that the core rotates from a minimum of 8 to a maximum of 17 times faster than the surface, confirming previous inversion results generated for other red giants (Deheuvels et al.), but we also estimate the variation of the angular velocity within the helium core with a spatial resolution of $0.001 R$ and verify the hypothesis of a sharp discontinuity in the inner stellar rotation. The results show that the entire core rotates rigidly and provide evidence for an angular velocity gradient around the base of the hydrogen-burning shell; however, we do not succeed in characterizing the rotational slope, due to the intrinsic limits of the applied techniques. The angular velocity, from the edge of the core, appears to decrease with increasing distance from the center, reaching an average value in the convective envelope of $68 \pm 22 \mathrm{nHz}$. We conclude that a set of data that includes only dipolar modes is sufficient to infer quite accurately the rotation of a red giant not only in the dense core but also, with a lower level of confidence, in part of the radiative region and in the convective envelope.
\end{abstract}

Key words: stars: AGB and post-AGB - stars: individual (KIC 4448777) - stars: interiors - stars: oscillations stars: rotation - stars: solar-type

\section{INTRODUCTION}

Stellar rotation is one of the fundamental processes governing stellar structure and evolution. The internal structure of a star at a given phase of its life is strongly affected by its history of angular momentum transport. Investigating the internal rotational profile of a star and reconstructing its evolution with time become crucial in achieving basic constraints on the mechanisms of angular momentum transport acting in the stellar interior during different phases of its evolution. In particular, physical processes that affect rotation and in turn are affected by rotation, such as convection, turbulent viscosity, meridional circulation, mixing of elements, internal gravity waves, dynamos, and magnetism, are at present not well understood and modeled with limited success (e.g., Marques et al. 2013; Cantiello et al. 2014).

Until fairly recently, rotation inside stars has been a largely unexplored field of research from an observational point of view. Over the past two decades helioseismology has changed this scenario, making it possible to measure the rotation profile in the Sun's interior through the measurement of the splittings of the oscillation frequencies, revealing a picture of the solar internal dynamics very different from previous theoretical predictions (see, e.g., Elsworth et al. 1995; Schou et al. 1998; Thompson et al. 2003). Contrary to what one could expect from the theory of angular momentum conservation, predicting a Sun with a core rotating much faster than the surface when only meridional circulation and classical hydrodynamic instabilities are invoked (e.g., Chaboyer et al. 1995), helioseismology shows an almost uniform rotation in the radiative interior and an angular velocity monotonically decreasing from the equator to high latitudes in the convective envelope. This strongly supports the idea that several powerful processes act to redistribute angular momentum in the interior, like for example magnetic torquing (e.g., Brun \& Zahn 2006) and internal gravity waves (e.g., Talon \& Charbonnel 2005).

The rotation breaks the spherical symmetry of the stellar structure and splits the frequency of each oscillation mode of harmonic degree $l$ into $2 l+1$ components, which appear as a multiplet in the power spectrum. Multiplets with a fixed radial order $n$ and harmonic degree $l$ are said to exhibit a frequency "splitting" defined by

$$
\delta \nu_{n, l, m}=\nu_{n, l, m}-\nu_{n, l, 0},
$$

somewhat analogous to the Zeeman effect on the degenerate energy levels of an atom, where $m$ is known as the azimuthal 
order. Under the hypothesis that the rotation of the star is sufficiently slow that effects of the centrifugal force can be neglected, the frequency separation between components of the multiplet is directly related to the angular velocity (Cowling \& Newing 1949).

In recent years spectacular asteroseismic results on data provided by the space missions CoRoT (Baglin et al. 2006) and Kepler (Borucki et al. 2010) have revolutionized the field. In particular, the Kepler satellite has provided photometric time series of unprecedented quality, cadence, and duration, supplying the basic conditions for studying the internal rotational profile and its temporal evolution in a large sample of stars, characterized by a wide range of masses and evolutionary stages.

In this context the detection of solar-like pulsations-excited by turbulent convection as in the Sun-in thousands of red giants, from the bottom to the tip of the red-giant branch (see, e.g., Mosser et al. 2013a; Stello et al. 2013) and to the asymptotic giant branch (Corsaro et al. 2012), appears particularly exciting. Red-giant stars are ideal asteroseismic targets for many reasons. Compared to the main-sequence stars, solar-like oscillations in red giants are easier to detect due to their higher pulsation amplitudes (Mosser et al. 2013b). What is more important, red-giant frequency spectra reveal mixed modes (see, e.g., Beck et al. 2011), which probe not only the outer layers, where they behave like acoustic modes, but also the deep radiative interior, where they propagate as gravity waves. Both the gravity-wave and the acoustic-wave propagation zones contribute, in various proportions, to the formation of mixed modes. The greatest contribution from the acoustic zone occurs for modes with frequency near the resonant frequency of the acoustic cavity. Mode inertias attain local minima at these frequencies.

Moreover, the red-giant phase represents a crucial step in the history of the stellar angular momentum distribution (Ceillier et al. 2013; Marques et al. 2013). When a star evolves off the relatively long and stable main sequence, its rotation starts evolving differently in the inner and outer parts, causing the formation of a sharp rotation gradient in the intermediate regions where hydrogen is still burning: assuming that the angular momentum is locally conserved, the contraction of the core causes its rotation to speed up in a relatively short timescale, while the outer layers slow down due to their expansion. The accurate determination of the rotational profiles in subgiants and red giants provides information on the mechanism of angular momentum transport, potentially leading to significant improvements in the modeling of stellar structure and evolution.

Recently, results based on measurements of the rotational splittings of dipole mixed modes have been reported in the literature (e.g., Beck et al. 2012, 2014; Deheuvels et al. 2012, 2014; Mosser et al. 2012b). Beck et al. (2012), based on highprecision measurements of rotational splittings provided by Kepler, found that the core in the red-giant stars is rotating faster than the upper layers. These results were confirmed by applying inversion techniques to rotational splittings by Deheuvels et al. (2012, 2014). Asteroseismology of a large sample of stars (Mosser et al. 2012b; Deheuvels et al. 2014) allowed researchers to clarify that the mean core rotation significantly slows down as stars ascend the red-giant branch.

Several theoretical investigations have explored the consequences of these recent results for internal angular momentum transport inside solar-like oscillating stars as they evolve (e.g., Ceillier et al. 2013; Marques et al. 2013; Tayar \& Pinsonneault 2013; Cantiello et al. 2014). These results show that the internal rotation rates, predicted by current theoretical models of subgiants and red giants, are at least 10 times higher than observations, suggesting the need to investigate more efficient mechanisms of angular momentum transport acting on the appropriate timescales during these phases of stellar evolution.

In this paper we analyze more than two years of Kepler observations of the red-giant star KIC 4448777 and identify 14 rotational splittings of mixed modes in order to characterize its internal rotational profile using different inversion techniques at first applied successfully to helioseismic data (e.g., Paternò et al. 1996; Thompson et al. 1996; Di Mauro \& Dziembowski 1998; Schou et al. 1998) and recently to data of more evolved stars (Deheuvels et al. 2012, 2014).

The paper is organized as follows: Section 2 reports the results of the spectroscopic analysis of the star aimed at the determination of its atmospheric parameters. Section 3 describes the method adopted to analyze the oscillation spectrum and identify the mode frequencies and the related splittings. Section 4 provides the basic formalism for performing the inversion, starting from the observed splittings and models of the star. Section 5 describes the evolutionary models constructed to best fit the atmospheric and asteroseismic constraints. Section 6 presents the details of the asteroseismic inversion carried out to infer the rotational profile of the star. In Section 7 we test the inversion techniques for the case of red giants and the capability of detecting the presence of rotational gradient in the deep interior of the star. In Section 8 the results obtained from the inversion techniques are compared with those obtained from other methods. Section 9 summarizes the results and draws conclusions.

\section{SPECTROSCOPIC ANALYSIS}

In order to properly characterize the star, six spectra of $1800 \mathrm{~s}$ integration time each were obtained with the HERMES spectrograph (Raskin et al. 2011), mounted on the $1.2 \mathrm{~m}$ MERCATOR telescope at La Palma. This highly efficient échelle spectrometer has a spectral resolution of $\mathrm{R}=86,000$, covering a spectral range from 380 to $900 \mathrm{~nm}$. The raw spectra were reduced with the instrument-specific pipeline and then averaged to a master spectrum. The signal-to-noise ratio was around 135 in the range from 500 to $550 \mathrm{~nm}$.

The determination of atmospheric parameters was based upon Fe I and Fe II lines, which are abundantly present in redgiant spectra. We used the local thermal equilibrium (LTE) Kurucz-Castelli atmosphere models (Castelli \& Kurucz 2004) combined with the LTE abundance calculation routine MOOG (version 2010 August) by C. Sneden. Fe lines were identified using VALD line lists (Kupka et al. 2000). For a detailed description of the different steps needed to determine atmospheric parameters, see, e.g., De Smedt et al. (2012). We selected Fe lines in the region of the master spectrum with the highest signal-to-noise ratio in the wavelength range between 500 and $680 \mathrm{~nm}$. The equivalent width $(\mathrm{EW})$ was calculated using direct integration and the abundance of each line was then computed by an iterative process where theoretically calculated EWs were matched to observed EWs. Due to the high metallicity, the spectrum of KIC 4448777 displays many blended lines. To avoid these blended lines in our selected Fe 
Table 1

KIC 4448777 Atmospheric Parameters

\begin{tabular}{lcccc}
\hline \hline$M_{V}$ & $T_{\text {eff }}(\mathrm{K})$ & $\log g(\mathrm{dex})$ & {$[\mathrm{Fe} / \mathrm{H}]$} & $v \sin i\left(\mathrm{~km} \mathrm{~s}^{-1}\right)$ \\
\hline $11.56^{\mathrm{a}}$ & $4750 \pm 250$ & $3.5 \pm 0.5$ & $0.23 \pm 0.12$ & $<5$ \\
\hline
\end{tabular}

Note.

${ }^{a}$ Kepler catalog.

line lists, we first calculated the theoretical EW of all available $\mathrm{Fe}_{\mathrm{I}}$ and $\mathrm{Fe}$ II lines in the wavelength range between 500 and $680 \mathrm{~nm}$. The theoretical EWs were then compared to the observed EWs to detect any possible blends. The atmospheric stellar parameters derived by the spectroscopic analysis are reported in Table 1 and are based upon the results from $46 \mathrm{Fe}$ I and $32 \mathrm{Fe}$ II lines.

We have also explored the possibility of deriving the surface rotation rate by following the method of García et al. (2014), but we have not found any signatures of spot modulation as evidence for an on-going magnetic field.

\section{TIME SERIES ANALYSIS AND FOURIER SPECTRUM}

For the asteroseismic analysis we have used near-continuous photometric time series obtained by Kepler in long-cadence mode (time sampling of 29.4 minutes). This light curve spans about 25 months corresponding to observing quarters Q0-9, providing a formal frequency resolution of $15 \mathrm{nHz}$. We used the so-called PDC-SAP (pre-data conditioning-simple aperture photometry) light curve (Jenkins et al. 2010) corrected for instrumental trends and discontinuities as described by García et al. (2011).

The power spectrum of the light curve shows a clear power excess in the range (180-260) $\mu \mathrm{Hz}$ (Figure 1) due to radial modes, with the comb-like pattern typical of the solar-like pmode oscillations, and non-radial modes, particularly those of spherical degree $l=1$, modulated by the mixing with g modes.

The initial analysis of the spectrum was done using the pipeline described in Huber et al. (2009). By this method we determined the frequency at maximum oscillation power $\nu_{\max }=(219.75 \pm 1.23) \mu \mathrm{Hz}$ and the so-called large frequency separation between modes with the same harmonic degree $\Delta \nu=(16.96 \pm 0.03) \mu \mathrm{Hz}$. The quoted uncertainties have been derived from analyzing 500 spectra generated by randomly perturbing the original spectrum, according to Huber et al. (2012). For the purposes of this paper it has been necessary, also, to identify the individual modes and measure their frequencies. This process, known as "peak bagging", is notoriously difficult to perform for red giants because of their complex frequency spectra, with modes of very different characteristics within narrow, sometimes even overlapping, frequency ranges. Mixed modes of different inertia have very different damping times and hence also different profiles in the frequency spectrum. Here we therefore used a combination of known methods tailored to our particular case, although we should mention that an automatic "one-fits-all" approach has recently been developed by Corsaro \& De Ridder (2014).

Four independent groups (simply called fitters) performed the fitting of the modes by using slightly different approaches:

1. The first team smoothed the power spectrum of the star to account for the intrinsic damping and re-excitation of the modes. They located the modes by two separate steps

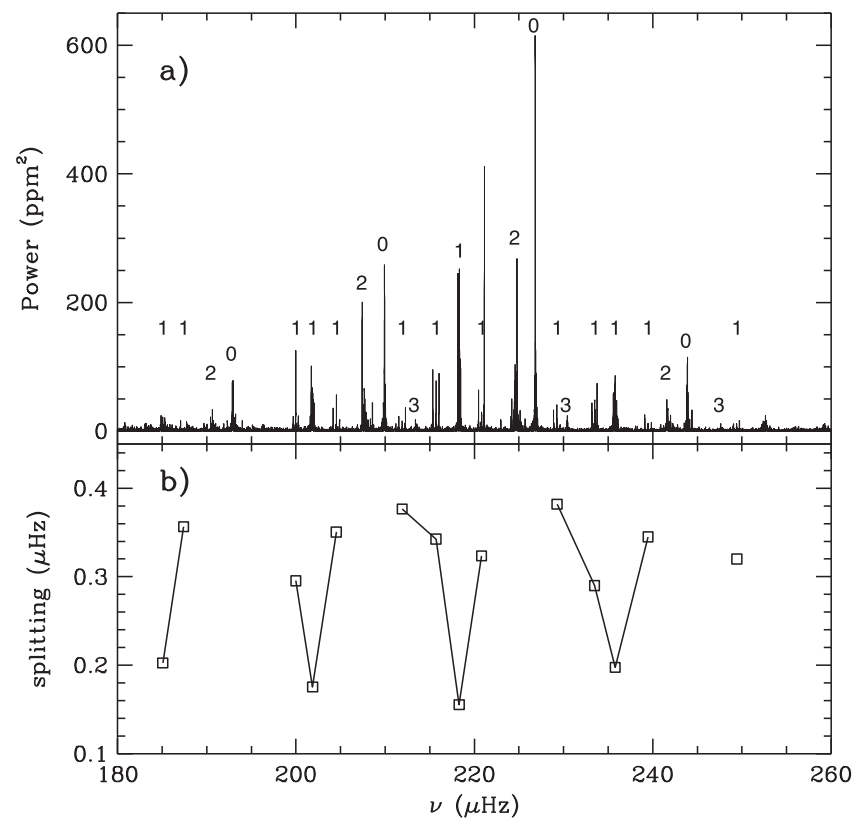

Figure 1. (a) Observed frequency spectrum of KIC 4448777. The harmonic degrees of the modes $(l=0,1,2,3)$ are indicated. Multiplets due to rotation are visible for $l=1$. (b) Observed rotational splittings for $l=1$ modes.

using a different level of smoothing in each. First, they heavily smoothed the power spectrum (by 13 independent bins in frequency) to detect the most damped modes including radial, quadrupole, and dipole modes with lower inertia, and then they smoothed less (by seven bins) to identify the dipole modes with higher inertia. In both cases the peaks were selected and associated with modes only if they were significant at the $99 \%$ level, setting the threshold according to the statistics for smoothed spectra, which takes into account the level of smoothing applied and the frequency range over which the modes have been searched (see, e.g., Chaplin et al. 2002). In addition, the "toy model" of Stello (2012) was used to locate a few extra dipole modes. This fitting was performed using the Bayesian Markov-chain Monte Carlo (MCMC) method by Handberg \& Campante (2011).

2. The second team extracted the frequencies of individual modes as the centroid of the unsmoothed power spectral density in narrow predefined windows, and checked for consistency by fitting Lorentzian profiles to a number of modes (Beck 2013).

3. The third team modeled the power spectrum with a sum of many Lorentzians, performed a global fit using a maximum likelihood estimator, and calculated the formal uncertainties from the inverse Hessian matrix (Mathur et al. 2013).

4. The fourth team derived proxies of the oscillation frequencies from a global fit based on global seismic parameters; all peaks with a height-to-background ratio larger than 8 were selected; radial modes and quadrupole modes were estimated from the fit of the large separation provided by the second-order asymptotic expansion for pressure modes (Mosser et al. 2011); the dipole modes were obtained with the asymptotic expansion for mixed modes (Mosser et al. 2012a) with rotational splittings derived with the method of Goupil et al. (2013). 
The final set of 58 individual mode frequencies, including the multiplets due to rotation for the $l=1$ modes, consists only of those frequencies detected by at least two of the fitters.

In order to obtain statistically consistent uncertainties for the mode frequencies we used the Bayesian MCMC fitting algorithm of Handberg \& Campante (2011) for the peak bagging. The algorithm allows simultaneous fitting of the stellar granulation signal (Mathur et al. 2011) and all oscillation modes, each represented by a Lorentzian profile. However, due to the complexity of the frequency spectrum of KIC 4448777 , we were not able to fit all modes simultaneously using a single method. In particular, the mixed modes with very high inertia and hence very long mode lifetimes have essentially undersampled frequency profiles in the spectrum. Fitting Lorentzian profiles to these modes is therefore unsuitable, and can easily lead the fitting algorithm astray.

We therefore treated the radial and quadrupole modes separately from the dipole modes. The radial and quadrupole modes were fitted as Lorentzian profiles using the MCMC approach. In this analysis we ignored any mixing of quadrupole modes and hence fit only one quadrupole mode per radial order.

For the dipole modes, which we were not able to fit as part of the MCMC method for the reasons explained above, we decided to adopt the initial frequencies, found from the smoothed spectrum by Team 1, as the final frequencies and the scatter among the four fitters as a proxy for uncertainties. As a sanity check for this approach we compared the uncertainties obtained by the MCMC fitting procedure of the radial modes with the scatter between different fitters of the same modes. We found that on average the fitter scatter is within $17 \%$ of the MCMC uncertainty, and all fitter scatter values are within a factor of two of the MCMC-derived uncertainty.

In the above analysis each dipole mode was detected separately, independently of the azimuthal order $m$. As in Beck et al. (2014), we noticed that the components with $m= \pm 1$ of a given triplet are not equally spaced from the central $m=0$ mode. In the framework of the perturbation theory, the splitting asymmetries correspond to second-order effects in the oscillation frequency that mainly account for the distortion caused by the centrifugal force. Here, the asymmetry is smaller in size and reasonably negligible, to a first approximation, when compared to the rotational splitting itself, ranging from $0.3 \%$ to-at most $-12 \%$ (with a mean value of $6 \%$ ) of the respective rotational splittings, with values comparable to the frequency uncertainties. In order to remove second-order perturbation effects, here we used the generalized rotational splitting expression (e.g., Goupil 2009):

$$
\delta \nu_{n, l}=\frac{\nu_{n, l, m}-\nu_{n, l,-m}}{2 m}
$$

The relative uncertainties have been calculated according to the general propagation formula for indirect measurements. Detailed investigation of the physical meaning of rotational splitting asymmetries and the possibility of deriving from them more stringent constraints on the internal rotational profile of oscillating stars have been the subject of different papers (see Suárez et al. 2010), but this is beyond the aim of the present work.

Table 2 lists the final set of frequencies together with their uncertainties, corresponding to the values obtained by the
MCMC fitting procedure for radial and quadrupole modes and to the scatter in the results from the four fitters for dipole modes, their spherical degree, and the rotational splittings for 14 dipole modes.

To measure the inclination of the star we used the above MCMC peak bagging algorithm, restricting the fit to the strongest dipole modes of $m=0$ and imposing equal spacing of the $m= \pm 1$ components. This calculation provided an inclination of $i=32.6_{-4.3}^{+5.0} \mathrm{deg}$.

As in Beck et al. (2012), the observed rotational splittings are not constant for consecutive dipole modes (see Figure 1(b) showing rotational splittings for the $l=1$ modes). Splittings are larger for modes with a higher inertia, which predominately probe the inner radiative interior. This shows that the deep interior of the star is rotating faster than the outer layers.

The identification of several dipole modes and the use of the method by Mosser et al. (2012a), based on the asymptotic relation, allowed us to estimate the asymptotic period spacing $\Delta \Pi_{1}=89.87 \pm 0.07 \mathrm{~s}$, which places this star on the lowluminosity red-giant branch in agreement with the evolutionary phase predicted by the value of the observed $\Delta \nu$ (Bedding et al. 2011; Mosser et al. 2012a; Stello et al. 2013).

A first estimate of the asteroseismic stellar mass and radius can be obtained from the observed $\Delta \nu$ and $\nu_{\max }$ together with the value of $T_{\text {eff }}$ (Kjeldsen \& Bedding 1995; Kallinger et al. 2010; Belkacem et al. 2011; Miglio et al. 2012). In particular, by using the scaling relation calibrated on solar values, we obtain $M_{\text {ast }} / M_{\odot}=1.12 \pm 0.09$ and $R_{\text {ast }} / \mathrm{R}_{\odot}$ $=4.13 \pm 0.11$. By using the scaling relation by Mosser et al. (2013a), calibrated on a large sample of observed solar-like stars, we obtain $M_{\text {ast }} / \mathrm{M}_{\odot}=1.02 \pm 0.05$ and $R_{\text {ast }} / \mathrm{R}_{\odot}$ $=3.97 \pm 0.06$. From the above values we can determine the asteroseismic surface gravity to be $\log g_{\text {ast }}=3.25 \pm 0.03$ dex. This value is in good agreement with that determined by the spectroscopic analysis (see Table 1).

\section{ASTEROSEISMIC INVERSION}

Asteroseismic inversion is a powerful tool that allows us to estimate the physical properties of stars by solving integral equations expressed in terms of the experimental data.

Previous experience acquired in helioseismology on inverting solar data represents a useful background for asteroseismic inversion. Earlier attempts at generalizing the standard helioseismic differential methods to find the structural differences between the observed star and a model have been applied to artificial data with encouraging results by Gough \& Kosovichev (1993), Roxburgh et al. (1998), and Berthomieu et al. (2001). More recently, Di Mauro (2004) was able to infer the internal structure of Procyon A below $0.3 R$ by inversion of real data comprising 55 low-degree $\mathrm{p}$ mode frequencies observed in the star. A general conclusion from these previous investigations is that the success of the inversion depends strongly on the number of observed frequencies and the accuracy with which the model represents the star.

Stellar inversions to infer the internal rotational profiles of stars were first applied to artificial data of moderately rotating stars such as $\delta$-Scuti stars (Goupil et al. 1996) and white dwarfs (Kawaler et al. 1999). Rotational inversion of simulated data of solar-like stars was studied by Lochard et al. (2005) for the case of a subgiant model representative of $\eta$ Boo. They showed that mixed modes can improve the inversion results on the internal 
Table 2

Measured Central Frequencies $(m=0)$ and Rotational Splittings for KIC 4448777

\begin{tabular}{|c|c|c|c|c|c|}
\hline$l$ & $\nu_{n, l}(\mu \mathrm{Hz})$ & $\delta \nu_{n, l}(\mu \mathrm{Hz})$ & $l$ & $\nu_{n, l}(\mu \mathrm{Hz})$ & $\delta \nu_{n, l}(\mu \mathrm{Hz})$ \\
\hline 0 & $159.842 \pm 0.014$ & n.a. ${ }^{\text {a }}$ & 2 & $174.005 \pm 0.043$ & $\ldots$ \\
\hline 0 & $176.277 \pm 0.018$ & n.a. & 2 & $190.623 \pm 0.034$ & $\cdots$ \\
\hline 0 & $192.907 \pm 0.016$ & n.a. & 2 & $207.551 \pm 0.026$ & $\ldots$ \\
\hline 0 & $209.929 \pm 0.014$ & n.a. & 2 & $224.646 \pm 0.011$ & $\ldots$ \\
\hline 0 & $226.831 \pm 0.014$ & n.a. & 2 & $241.630 \pm 0.022$ & $\cdots$ \\
\hline 0 & $243.879 \pm 0.013$ & n.a. & 3 & $213.443 \pm 0.015$ & $\ldots$ \\
\hline 0 & $261.215 \pm 0.034$ & n.a. & 3 & $230.423 \pm 0.011$ & $\ldots$ \\
\hline 1 & $167.061 \pm 0.011$ & $\ldots$ & 3 & $247.600 \pm 0.017$ & $\cdots$ \\
\hline 1 & $185.069 \pm 0.011^{\mathrm{b}}$ & $0.2025 \pm 0.0078$ & $\ldots$ & $\ldots$ & $\ldots$ \\
\hline 1 & $187.402 \pm 0.011^{\mathrm{b}}$ & $0.3565 \pm 0.0078$ & $\ldots$ & $\ldots$ & $\ldots$ \\
\hline 1 & $199.986 \pm 0.011^{\mathrm{b}}$ & $0.2955 \pm 0.0078$ & $\ldots$ & $\ldots$ & $\cdots$ \\
\hline 1 & $201.864 \pm 0.011^{\mathrm{b}}$ & $0.1755 \pm 0.0078$ & $\ldots$ & $\ldots$ & $\ldots$ \\
\hline 1 & $204.528 \pm 0.011^{\mathrm{b}}$ & $0.3505 \pm 0.0078$ & $\ldots$ & $\ldots$ & $\ldots$ \\
\hline 1 & $208.571 \pm 0.011^{\mathrm{b}}$ & $\ldots$ & $\ldots$ & $\ldots$ & $\ldots$ \\
\hline 1 & $211.913 \pm 0.011^{\mathrm{b}}$ & $0.3765 \pm 0.0078$ & $\ldots$ & $\ldots$ & $\ldots$ \\
\hline 1 & $215.699 \pm 0.015^{\mathrm{b}}$ & $0.3425 \pm 0.0078$ & $\ldots$ & $\ldots$ & $\cdots$ \\
\hline 1 & $218.299 \pm 0.017^{\mathrm{b}}$ & $0.1555 \pm 0.0078$ & $\ldots$ & $\ldots$ & $\cdots$ \\
\hline 1 & $220.814 \pm 0.018^{\mathrm{b}}$ & $0.3235 \pm 0.0078$ & $\ldots$ & $\ldots$ & $\ldots$ \\
\hline 1 & $229.276 \pm 0.011^{\mathrm{b}}$ & $0.3820 \pm 0.0160$ & $\ldots$ & $\ldots$ & $\ldots$ \\
\hline 1 & $233.481 \pm 0.011^{\mathrm{b}}$ & $0.2900 \pm 0.0078$ & $\ldots$ & $\ldots$ & $\ldots$ \\
\hline 1 & $235.783 \pm 0.011^{\mathrm{b}}$ & $0.1975 \pm 0.0140$ & $\ldots$ & $\ldots$ & $\ldots$ \\
\hline 1 & $239.463 \pm 0.011^{\mathrm{b}}$ & $0.3450 \pm 0.0160$ & $\ldots$ & $\cdots$ & $\cdots$ \\
\hline 1 & $244.385 \pm 0.011^{\mathrm{b}}$ & $\ldots$ & $\ldots$ & $\ldots$ & $\ldots$ \\
\hline 1 & $249.417 \pm 0.011^{\mathrm{b}}$ & $0.3200 \pm 0.0210$ & $\ldots$ & $\ldots$ & $\ldots$ \\
\hline 1 & $252.377 \pm 0.021^{\mathrm{b}}$ & $\ldots$ & $\ldots$ & $\ldots$ & $\ldots$ \\
\hline 1 & $252.661 \pm 0.016^{\mathrm{b}}$ & $\ldots$ & $\ldots$ & $\ldots$ & $\ldots$ \\
\hline
\end{tabular}

Notes.

${ }^{a}$ n.a. = not applicable.

${ }^{\mathrm{b}}$ Uncertainties for the $l=1$ modes correspond to the scatter in the results from the four fitters (see text).

rotation of the star, while data limited to pure $l=1,2 \mathrm{p}$ modes are not sufficient to provide reliable solutions. Indeed, striking results on rotation have been obtained by Deheuvels et al. (2012), who performed a detailed modeling of the red-giant star KIC 7341231, located at the bottom of the red-giant branch. They performed an inversion of the internal stellar rotation profile based on observed rotational splittings of 18 mixed modes. They found that the core is rotating at least five times faster than the envelope. More recently Deheuvels et al. (2014) applied their techniques to six subgiants and low-luminosity red giants.

The internal rotation of KIC 4448777 can be quantified by inverting the following equation (Gough 1981), obtained by applying a standard perturbation theory to the eigenfrequencies, in the hypothesis of slow rotation:

$$
\delta \nu_{n, l}=\int_{0}^{R} \mathcal{K}_{n, l}(r) \frac{\Omega(r)}{2 \pi} d r+\epsilon_{n, l}
$$

where $\delta \nu_{n, l}$ is the adopted set of splittings, $\Omega(r)$ is the internal rotation assumed to be a function of only the radial coordinate, $\epsilon_{n, l}$ are the uncertainties in the measured splittings, and $\mathcal{K}_{n, l}(r)$ are the mode kernel functions calculated on the unperturbed eigenfunctions for the modes $(n, l)$ of the best model of the star:

$$
\mathcal{K}_{n, l}(r)=\frac{1}{I}\left[\xi_{r}^{2}+l(l+1) \xi_{h}^{2}-2 \xi_{r} \xi_{h}-\xi_{h}^{2}\right] \rho r^{2},
$$

where $\xi_{r}$ and $\xi_{h}$ are the radial and horizontal components of the displacement vector respectively, $\rho$ is the density, and $R$ is the photospheric stellar radius, while the inertia is given by

$$
I=\int_{0}^{R}\left[\xi_{r}^{2}+l(l+1) \xi_{h}^{2}\right] \rho r^{2} d r .
$$

The properties of the inversion depend both on the mode selection $i \equiv(n, l)$ and on the observational uncertainties $\epsilon_{i}$ that characterize the mode set $i=1, \ldots, N$ to be inverted.

The main difficulty in solving Equation (3) for $\Omega(r)$ arises from the fact that the inversion is an ill-posed problem: the observed splittings constitute a finite and quite small set of data and the uncertainties in the observations prevent the solution from being determined with certainty. Thus, an appropriate choice for a suitable inversion technique is the first important step during an asteroseismic inverse analysis.

\subsection{Inversion Procedure}

There are two important classes of methods for obtaining estimates of $\Omega(r)$ from Equation (3): the optimally localized averaging (OLA) method, based on the original idea of Backus \& Gilbert (1970), and the regularized least-squares fitting method (Phillips 1962; Tikhonov 1963). Both methods give linear estimates of the function $\Omega(r)$ with results generally in agreement, as was demonstrated by Christensen-Dalsgaard et al. (1990), Sekii (1997), and Deheuvels et al. (2012).

Here we study and apply the OLA method and its variant form, which allows us to estimate a localized weighted average of angular velocity $\bar{\Omega}\left(r_{0}\right)$ at selected target radii $\left\{r_{0}\right\}$ by means 
of a linear combination of all the data:

$$
\frac{\bar{\Omega}\left(r_{0}\right)}{2 \pi}=\sum_{i=1}^{N} c_{i}\left(r_{0}\right) \delta \nu_{i}=\int_{0}^{R} K\left(r_{0}, r\right) \frac{\Omega(r)}{2 \pi} d r,
$$

where $c_{i}\left(r_{0}\right)$ are the inversion coefficients and

$$
K\left(r_{0}, r\right)=\sum_{i=1}^{N} c_{i}\left(r_{0}\right) \mathcal{K}_{i}(r)
$$

are the averaging kernels. Here we adapted the code, developed for solar rotation in Paternò et al. (1996), to be applied to any evolutionary phase.

Because of the ill-conditioned nature of the inversion problem, it is necessary to introduce a regularization procedure. By varying a trade-off parameter $\mu$, we look for the coefficients $c_{i}\left(r_{0}\right)$ that minimize the propagation of the uncertainties and the spread of the kernels:

$$
\int_{0}^{R} J\left(r_{0}, r\right) K\left(r_{0}, r\right)^{2} d r+\frac{\mu}{\mu_{0}} \sum_{i=1}^{N} \epsilon_{i}^{2} c_{i}^{2}\left(r_{0}\right),
$$

where

$$
\mu_{0}=\frac{1}{N} \sum_{i=1}^{N} \epsilon_{i}^{2}
$$

assuming that

$$
\int_{0}^{R} K\left(r_{0}, r\right) d r=1 .
$$

$J\left(r_{0}, r\right)$ is a weight function, small near $r_{0}$ and large elsewhere, which has been assumed to be

$$
J\left(r_{0}, r\right)=12\left(r-r_{0}\right)^{2} / R,
$$

and is designed to build averaging kernels as close as possible to a Dirac function centered at $r_{0}$. The minimization of Equation (8) is equivalent to solving a set of $N$ linear equations for $c_{i}$. The uncertainties of the solutions are the standard deviations calculated in the following way:

$$
\sigma\left[\frac{\bar{\Omega}\left(r_{0}\right)}{2 \pi}\right]=\left[\sum_{i=1}^{N} c_{i}^{2}\left(r_{0}\right) \epsilon_{i}^{2}\right]^{1 / 2} .
$$

The center of mass of the averaging kernels is

$$
\bar{r}\left(r_{0}\right)=\int_{0}^{R} r K\left(r_{0}, r\right) d r .
$$

We also considered the method in the variant form, as described in Pijpers \& Thompson (1992), known as subtractive optimally localized averaging (SOLA), making attempts to fit the averaging kernel to a Gaussian function $G\left(r_{0}, r\right)$ of an appropriate width, centered at the target radius (Di Mauro \& Dziembowski 1998). The two parameters - the width of the Gaussian target function and the trade-off parameter-are tuned to find an acceptable matching of the averaging kernel to its target function and also to ensure an acceptable small error on the result from the propagation of the measurement errors. Therefore, the coefficients are determined by minimizing the following:

$$
\int_{0}^{R} R\left[K\left(r_{0}, r\right)-G\left(r_{0}, r\right)\right]^{2} d r+\frac{\mu}{\mu_{0}} \sum_{i=1}^{N} \epsilon_{i}^{2} c_{i}^{2}\left(r_{0}\right),
$$

where

$$
G\left(r_{0}, r\right)=\frac{1}{\sqrt{2 \pi \sigma^{2}}} \exp ^{-\left|r-r_{0}\right|^{2} / 2 \sigma^{2}}
$$

and $\sigma$ is chosen to fix the width of the Gaussian function.

\section{EVOLUTIONARY MODELS OF KIC 4448777}

We first need to construct a best fitting model of the star that satisfies all the observational constraints in order to quantify the internal rotation and to understand the relation between the observed rotational splittings and how sensitive each mode is to the different regions of that model. The theoretical structure models have been calculated by using the ASTEC evolution code (Christensen-Dalsgaard 2008a), spanning the parameter space given in Table 1 and following the procedure described in Di Mauro et al. (2011).

The input physics for the evolution calculations included the OPAL 2005 equation of state (Rogers \& Nayvonov 2002), OPAL opacities (Iglesias \& Rogers 1996), and the NACRE nuclear reaction rates (Angulo et al. 1999). Convection was treated according to the mixing-length formalism (MLT, Böhm-Vitense 1958) and defined through the parameter $\alpha=\ell / H_{p}$, where $H_{p}$ is the pressure scale height and $\alpha$ is varied from 1.6 to 1.8. The initial heavy-element mass fraction $Z_{i}$ has been calculated from the iron abundance given in Table 1 using the relation $[\mathrm{Fe} / \mathrm{H}]=\log (Z / X)-\log (Z / X)_{\odot}$, where $Z / X$ is the value at the stellar surface, and the solar value was taken to be $(Z / X)_{\odot}=0.0245$ (Grevesse \& Noels 1993). Thus, we used $Z / X=0.04 \pm 0.01$ in the modeling.

The resulting evolutionary tracks are characterized by the input stellar mass $M$, the initial chemical composition, and a mixing-length parameter. For the models with values of $T_{\text {eff }}$ and $\log g$ consistent with the spectroscopic observed values, we calculated the adiabatic oscillation frequencies using the ADIPLS code (Christensen-Dalsgaard 2008b). We applied the surface effect correction following the approach proposed by Kjeldsen et al. (2008) and using the prescription of Brandão et al. (2011), which takes into account that modes with high inertia suffer a smaller surface effect than do $\mathrm{p}$ modes. The correction applied to all calculated frequencies is then of the form:

$$
\nu_{n, l}^{\bmod }=\nu_{n, l}+a \frac{1}{Q_{n, l}}\left(\frac{\nu_{n, l}}{\nu_{0}}\right)^{b}
$$

where $\nu_{n, l}^{\text {mod }}$ are the corrected frequencies, $Q_{n, l}$ is the inertia of the given mode normalized by the inertia of a radial mode of the same frequency, obtained by interpolation, $\nu_{n, l}$ are the bestmodel frequencies, $\nu_{0}$ is a constant frequency, usually chosen to be the frequency at maximum oscillation power, $a$ is the amplitude of the correction at $\nu_{0}$, and $b$ is the exponent, assumed to be 4.90 like the one calculated for the solar frequencies by Kjeldsen et al. (2008).

The results of the fits between the observed star and the models were evaluated according to the total $\chi^{2}$ between the observed $\nu_{i}^{\text {obs }}$ and calculated $\nu_{i}^{\text {mod }}$ values of the individual oscillation frequencies as

$$
\chi^{2}=\frac{1}{N} \sum_{1}^{N}\left(\frac{\nu_{i}^{\mathrm{obs}}-\nu_{i}^{\mathrm{mod}}}{\epsilon_{i}}\right)^{2},
$$

where $\epsilon_{i}$ are the uncertainties on the observed frequencies. 
Table 3

Main Parameters for KIC 4448777 and for the Best Fitting Models

\begin{tabular}{lccc}
\hline \hline & KIC 4448777 & Model 1 & Model 2 \\
\hline$M / \mathrm{M}_{\odot}$ & $1.02 \pm 0.05^{\mathrm{a}}$ & 1.02 & 1.13 \\
Age $(\mathrm{Gyr})$ & $\ldots$ & 8.30 & 7.24 \\
$T_{\text {eff }}(\mathrm{K})$ & $4750 \pm 250^{\mathrm{b}}$ & 4800 & 4735 \\
$\log g(\mathrm{dex})$ & $3.5 \pm 0.5^{\mathrm{b}}$ & 3.26 & 3.27 \\
$R / \mathrm{R}_{\odot}$ & $3.97 \pm 0.06^{\mathrm{b}}$ & 3.94 & 4.08 \\
$L / \mathrm{L}_{\odot}$ & $\ldots$ & 7.39 & 7.22 \\
$Z_{i}$ & $\ldots$ & 0.015 & 0.022 \\
$X_{i}$ & $\ldots$ & 0.69 & 0.69 \\
{$[\mathrm{Fe} / \mathrm{H}]$} & $0.23 \pm 0.12^{\mathrm{b}}$ & -0.04 & 0.13 \\
$r_{c z} / R$ & $\ldots$ & 0.15 & 0.14 \\
$\alpha_{\mathrm{MLT}}$ & $\ldots$ & 1.80 & 1.80 \\
$\Delta \nu(\mu \mathrm{Hz})$ & $16.96 \pm 0.03$ & 16.97 & 16.93 \\
\hline
\end{tabular}

Notes. $M / \mathrm{M}_{\odot}$ is the mass of the star, $T_{\text {eff }}$ is the effective temperature, $\log g$ is the surface gravity, $R / \mathrm{R}_{\odot}$ is the surface radius, $L / \mathrm{L}_{\odot}$ is the luminosity, $Z_{i}$ is the initial metallicity, $X_{i}$ is the initial hydrogen abundance, $[\mathrm{Fe} / \mathrm{H}]$ is the iron abundance, $r_{c z}$ is the location of the base of the convective region, $\alpha_{\mathrm{MLT}}$ is the mixing-length parameter, and $\Delta \nu$ is the large separation obtained by linearly fitting the radial-mode frequencies.

${ }^{a}$ Calculated by using asteroseismic relations (Section 3).

${ }^{\mathrm{b}}$ Determined by spectroscopic observations (Section 2).

In Table 3 we give a comprehensive set of stellar properties for the two best fitting models compared with observations of KIC 4448777.

Figure 2 shows evolutionary tracks plotted in a HertzsprungRussell diagram for the two best-fitting models. The location of the star in the H-R diagram identifies KIC 4448777 as being at the beginning of the ascending red-giant branch. It has a small degenerate helium core, having exhausted its central hydrogen, and it is in the shell-hydrogen-burning phase. The hydrogen abundance as a function of the fractional mass and radius plotted for one of the selected models of KIC 4448777 shows the extent of the core with a radius $r_{c}=0.01 R$ and the location of the base of the convective zone (Figure 3). The outer convective zone appears to be quite deep, reaching about $r_{c z} \simeq 0.15 R$. It can be noticed that Model 2, during the mainsequence phase, develops a convective core, which lasts almost to the hydrogen exhaustion at the center. The higher metallicity of Model 2, in comparison to Model 1, leads to a high opacity and therefore one would expect a lower luminosity and no convective core in this evolutionary phase. However, in Model 2, the quite low hydrogen abundance determining a higher mean molecular weight acts to increase the luminosity, pushing again to develop a convective core.

As shown in the propagation diagram obtained for Model 1 in Figure 4, the huge difference in density between the core region and the convective envelope causes a large value of the buoyancy frequency in the core, determining well-defined acoustic and gravity-wave cavities, with modest interaction between $\mathrm{p}$ and $\mathrm{g}$ modes.

Figure 5 shows the échelle diagram obtained for the two models. The results show, as explained in previous sections, that the observed modes are $l=0$ pure acoustic modes, and $l=1,2,3 \mathrm{~g}-\mathrm{p}$ mixed modes. Several non-radial mixed modes have a very low inertia, hence they propagate in the lowdensity region, namely the acoustic cavity, and behave like $\mathrm{p}$ modes. Most of the $l=1$ mixed modes have a quite high inertia, which means that they propagate in the gravity-wave cavity in the high-density region, although the mixing with a $p$

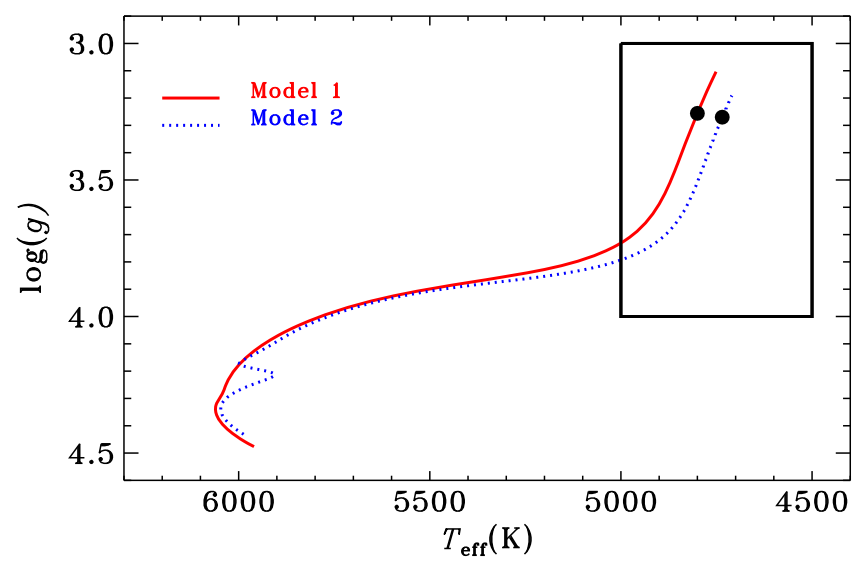

Figure 2. Evolutionary tracks plotted in an H-R diagram. Black dots indicate two models that best reproduce the observations. The rectangle indicates the $1 \sigma$ error box of the observed $\log g$ and $T_{\text {eff. }}$.

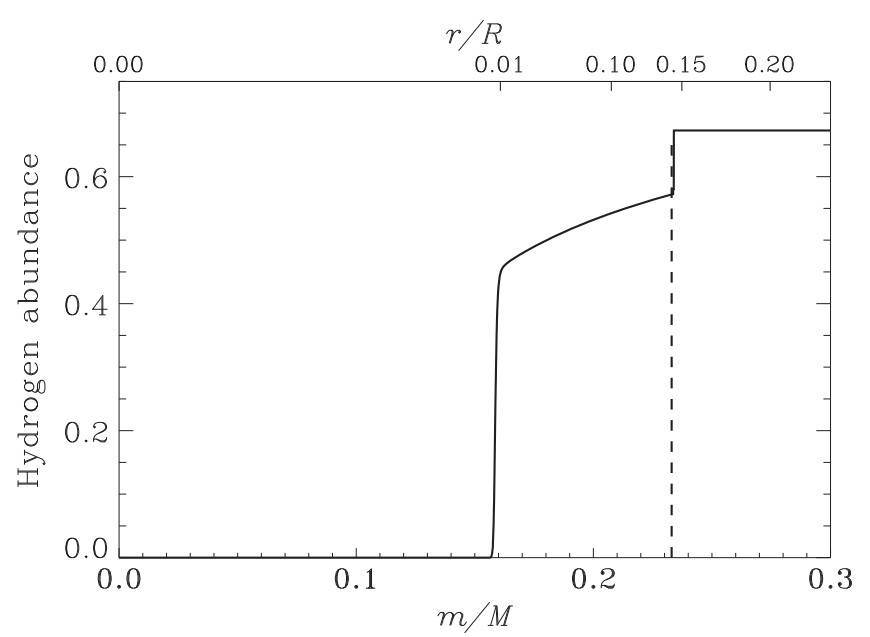

Figure 3. Hydrogen content in Model 2 of KIC 4448777. The base of the convective envelope located at $m_{c z}=0.23 M$ and $r_{c z}=0.14 R$ is shown by the dashed line.

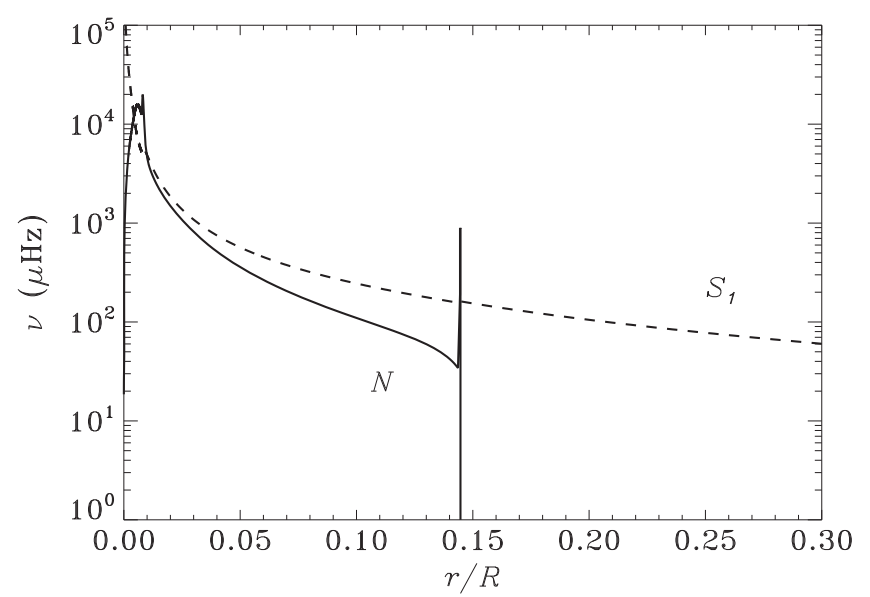

Figure 4. Propagation diagram from the center to $r=0.3 R$ for Model 1. The solid black line represents the buoyancy frequency $N$. The dashed line represents the Lamb frequency $S_{l}$ for $l=1$.

mode enhances their amplitude and hence ensures that they can be observed at the surface. In the échelle diagram these gravitydominated modes evidently depart from the regular solar-like pattern. 

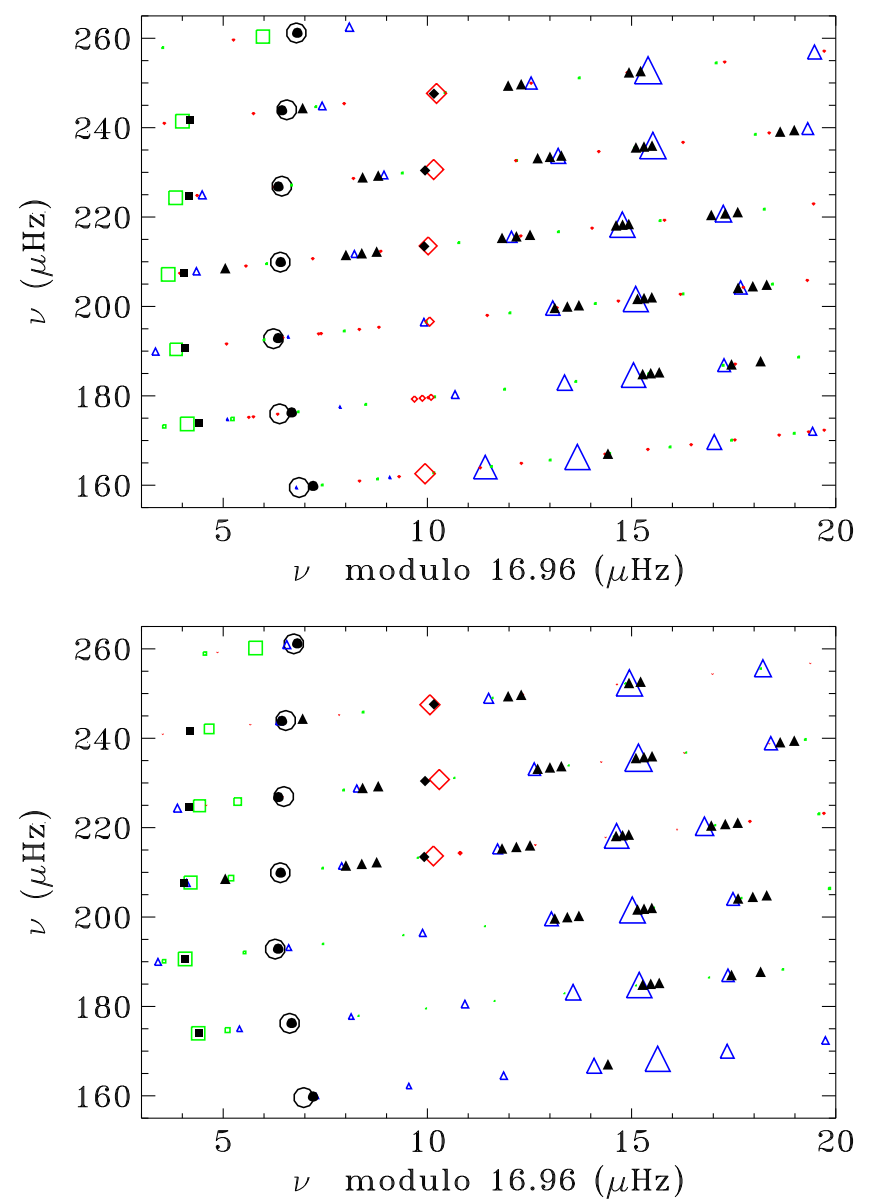

Figure 5. Échelle diagrams for Models 1 (upper panel) and 2 (lower panel) of Table 3. The filled symbols show the observed frequencies. The open symbols show computed frequencies. Circles are used for modes with $l=0$, triangles for $l=1$, squares for $l=2$, and diamonds for $l=3$. Observed splitting of $l=1$ modes can be seen as triplets or doublets of black triangles. The size of the open symbols indicates the relative surface amplitude of oscillation of the modes.

We found that there is an agreement between observed and theoretical frequencies of the two selected models, within $4 \sigma$ errors and with $\chi^{2}=45$ for Model 1 and $\chi^{2}=61$ for Model 2; however, we notice that Model 2 best reproduces the spectroscopic observation of the iron abundance.

\section{RESULTS OF THE ASTEROSEISMIC INVERSIONS}

Once the best model has been selected, it is then possible to invert Equation (3) following the procedure described in Section 4 . For this we used the 14 rotational splittings of the dipole modes given in Table 2.

Kernels calculated for Models 1 and 2, corresponding to two observed modes with different inertia, are shown as an example in Figure 6 (see also Goupil et al. 1996). It is interesting to notice that kernels calculated for the two different models, but corresponding to the same frequency, show similar amplitudes in the interior.

The inferred rotation rate obtained by applying the OLA technique for the two models is shown in Figure 7, where the points indicate the angular velocity against the selected target radii $\left\{r_{0}\right\}$. The radial spatial resolution is the interquartile range of the averaging kernels and gives a measure of the localization of the solution. In the probed regions the distance between the center of mass and the target location is smaller than the width of the averaging kernel (see Equation (13)).

To show more clearly the errors in the inferred internal rotation, the vertical bars are $2 \sigma, \sigma$ being the standard deviation given by Equation (10). Different trade-off parameters have been used with $\mu=0-10$ to try to better localize the kernels. A good compromise between localization and error magnification in the solution has been obtained using $\mu=0.001$ for both Model 1 and Model 2. The inversion parameter has been chosen by inverting a known simple rotational profile.

We were able to estimate the variation of the angular velocity with the radius in the inner interior with a spatial resolution of $\Delta r=0.001 R$, thanks to the very localized averaging kernels at different radii. Figure 8 shows OLA averaging kernels localized at several target radii $r_{0}$ obtained with a trade-off parameter $\mu=0.001$ for the inversion given in Figure 7 by using Model 2 .

We find an angular velocity in the core at $r=0.005 \mathrm{R}_{\odot}$ of $\Omega_{c \text { OLA }} / 2 \pi=749 \pm 11 \mathrm{nHz}$ with Model 1 , in good agreement with the value obtained with Model 2 which is $\Omega_{c \text { OLA }} / 2 \pi=744 \pm 12 \mathrm{nHz}$. The rotation appears to be constant inside the core and decreases smoothly from the edge of the helium core through the hydrogen-burning shell with increasing radius. In Figure 9 we plot the OLA cumulative integrals of the averaging kernels centered at different locations in the inner interior, to show in which region of the star the solutions are most sensitive. The cumulative kernels corresponding to solutions centered below and above the H-burning shell look quite similar. The leakage from the core explains the reason why the OLA results show an almost constant rotation in the He core and the H-burning shell.

We note that it is not possible to find localized solutions for $r_{0}>0.01 R$. Attempts to concentrate solutions above this point return averaging kernels that suffer from very large leakage from both the deep core and the superficial layers, as shown in Figure 8.

However, due to the p-mode contributions of certain modes considered, some reliable results can be found above $0.9 R$, although with fairly low weight as shown by the kernel centered around $r_{0}=0.98 R$ of Figure 8 . The angular velocity reaches a mean value in the convective envelope of $\Omega_{s \text { OLA }} / 2 \pi=68 \pm 22 \mathrm{nHz}$ with Model 1, in good agreement with $\Omega_{s}$ OLA $/ 2 \pi=60 \pm 14 \mathrm{nHz}$ obtained with Model 2 .

The angular velocity value below the surface and the significance of this result can be investigated by considering the cumulative integral of the averaging kernels $\int_{0}^{r} K\left(r_{0}, r\right) d r$, in order to understand where the kernels are most sensitive inside the star. Figure 10 shows that the surface averaging kernels provide a weighted average of the angular velocity of the layers $r>0.2 R$, in most of the convective envelope, and not an estimate of the rotation at the surface. This is due to the fact that the eigenfunctions of the modes considered here are too similar to one another to build averaging kernels localized at different radii in the acoustic cavity.

Moreover, Figure 10 shows that the present set of data does not allow us to appreciate the difference between the cumulative integral of the surface kernels calculated for the two models, hence the results obtained at the surface do not depend on the stellar model chosen. The detection of a larger number of modes trapped in the convective envelope would have made it possible to study the upper layers with a higher level of confidence. 

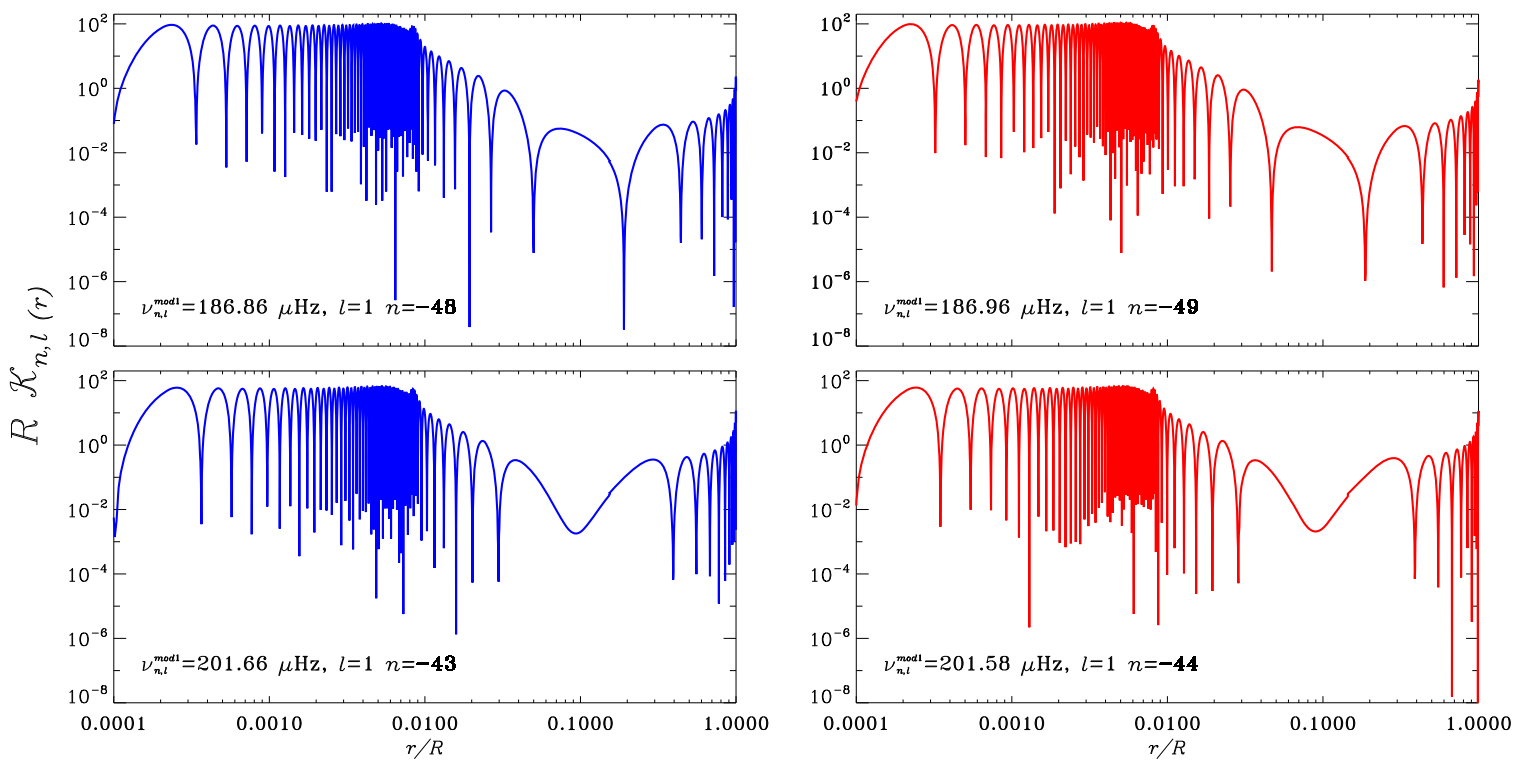

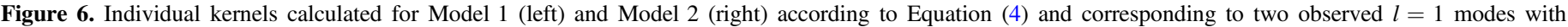

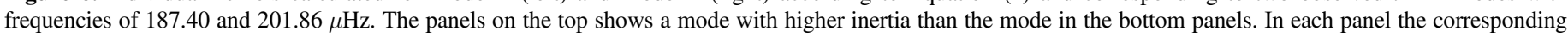
theoretical oscillation frequency, harmonic degree $l$, and radial order $n$ are indicated.

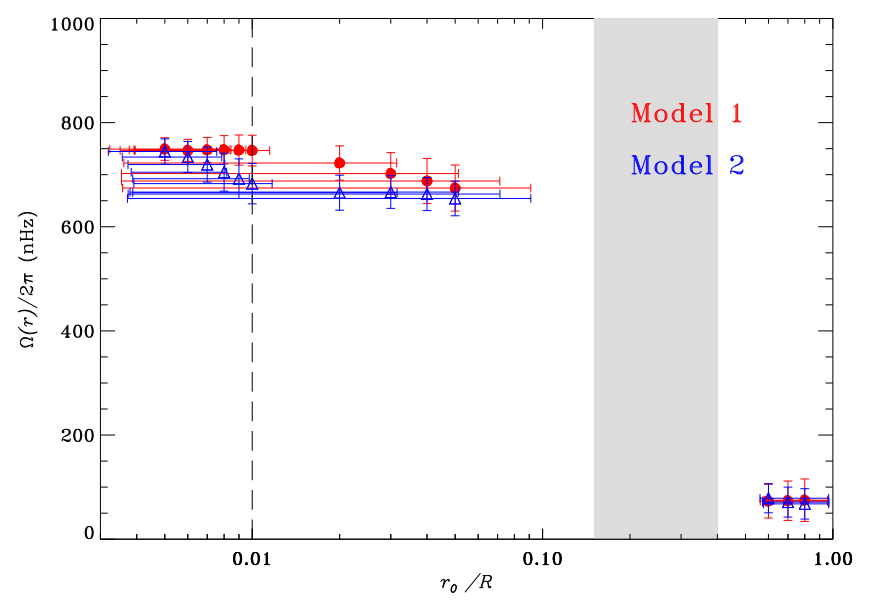

Figure 7. Internal rotation of KIC 4448777 at different depths as obtained by the OLA inversion based on the two best-fitting models. Vertical error bars are two standard deviations. The dashed line indicates the location of the inner edge of the H-burning shell. The shaded area indicates the region inside the star in which it was not possible to determine any solutions.

The solutions inferred from the SOLA method, plotted against the target radius, are shown in Figure 11. The radial resolution is equal to the width of the target Gaussian kernels, while the uncertainty in the solutions is plotted as two standard deviations, like for the OLA results. The values obtained for the angular velocity in the core at $r=0.004 R$ are $\Omega_{c \text { SOLA }} / 2 \pi=754 \pm 12 \mathrm{nHz}$ with Model 1 and $\Omega_{c \text { SOLA }} / 2 \pi=743 \pm 13 \mathrm{nHz}$ with Model 2, which are in good agreement with the values obtained by the OLA method.

On the other hand, and differing from the OLA results, above $r=0.01 R$ the angular velocity appears to drop rapidly, indicating an almost constant rotation from the edge of the core to the surface.

The SOLA technique produced reliable results only for $r<0.01 R$, due to the fact that we failed to fit the averaging kernels to the Gaussian target function, as required by the method. Figure 12 shows averaging kernels and Gaussian target functions for the solutions plotted in Figure 11. Here we used a trade-off parameter $\mu=1$, but we notice that the solutions appear to be sensitive to small changes in the tradeoff parameter $(0<\mu<10)$ only below the photosphere, with variation up to $20 \%$ in the results. The solutions in the core are not sensitive to the same changes of $\mu$ and the averaging kernels remain well localized. It is clear that only solutions related to averaging kernels that are well localized and close to the target Gaussian functions can be considered reliable.

In Figure 13 we plot the SOLA cumulative integrals of the averaging kernels corresponding to solutions at different locations in the interior. We find that the core cumulative kernel is very well localized, and the cumulative kernel for the solution at $r=0.01 R$ is localized with a percentage of $70 \%$, although contaminated from the layers of the convective envelope. Cumulative kernels for solutions with $r>0.01 R$ appear to be sensitive to the radiative region for a percentage that is quickly decreasing with increasing target radii, while the contamination from the outer layers appear high. Thus, we can conclude that while the OLA solutions are strongly affected by the core (Figure 9), the SOLA solutions appear more polluted by the signal from the surface layers. Nevertheless, the averaging kernel and cumulative kernel for the SOLA solution at $r=0.01 R$ turn out to be better localized than the OLA solution, indicating that the decrease occurring around the base of the H-burning shell is reliable.

The angular velocity below the surface at $r_{0}=0.85 R$ with the SOLA method turns out to be $\Omega_{s}$ SOLA $/ 2 \pi=28 \pm 14 \mathrm{nHz}$ with Model 1 and $\Omega_{s}$ Sola $/ \pi=11 \pm 16 \mathrm{nHz}$ with Model 2 . We can compare the surface cumulative integrals of the averaging kernels $\int_{0}^{r} K\left(r_{0}, r\right) d r$ as obtained for the two inversion methods and plotted in Figures 10 and 14. We found that the cumulative kernel integral for the near-surface SOLA inversion appears marginally contaminated by the kernels of the regions $r<0.2 R$, but the results can still be considered in good agreement with the OLA ones. In the SOLA averaging kernels it was not possible to suppress efficiently the strong 

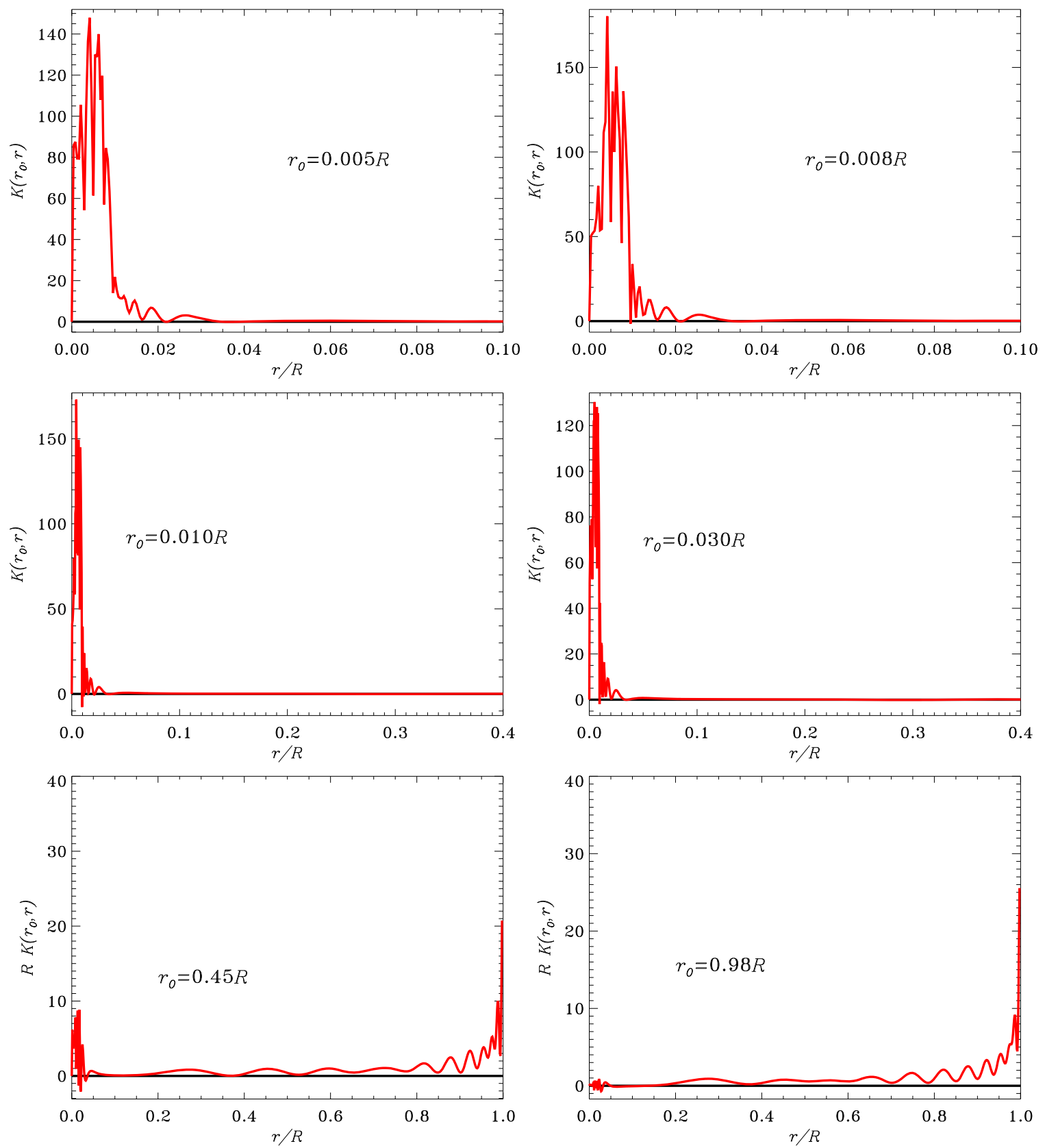

Figure 8. OLA averaging kernels localized at several target radii $r_{0}$ obtained with a trade-off parameter $\mu=0.001$ for the inversion given in Figure 7 by using Model 2.

signal of the modes concentrated in the core. We conclude that the angular velocity value obtained at the surface by applying the SOLA method represents a weighted average of the angular velocity of the entire interior. As a consequence, we think that, in this case, the OLA result should be preferred as a probe of the rotation in the convective envelope.

\section{A STRONG ROTATIONAL GRADIENT IN THE CORE?}

The results obtained in Section 6 raise the question about the possibility of the existence of a sharp gradient in the rotational profile localized at the edge of the core. Evolution of theoretical models that assumes conservation of angular momentum of the stellar interior predicts that, during the post-main-sequence phase, a sharp rotation gradient localized near the H-burning shell should form between a fast-spinning core and a slowly rotating envelope (see, e.g., Ceillier et al. 2013; Marques et al. 2013). However, if an instantaneous angular momentum transport mechanism is at work, the whole star should rotate as a solid body. The general understanding is that the actual stellar rotational picture should be something in between.

The occurrence of a sharp rotation gradient in post-mainsequence stars has been already investigated by other authors (see, e.g., Deheuvels et al. 2014), with no possibility to get a definitive conclusion.

In order to understand the differences in the inversion results at the edge of the core obtained by using the OLA and the SOLA methods, we tested both techniques by trying to recover simple input rotational profiles by computing and inverting artificial rotational splittings. In order to accomplish this task we used the forward seismological approach as described in $\mathrm{Di}$ 


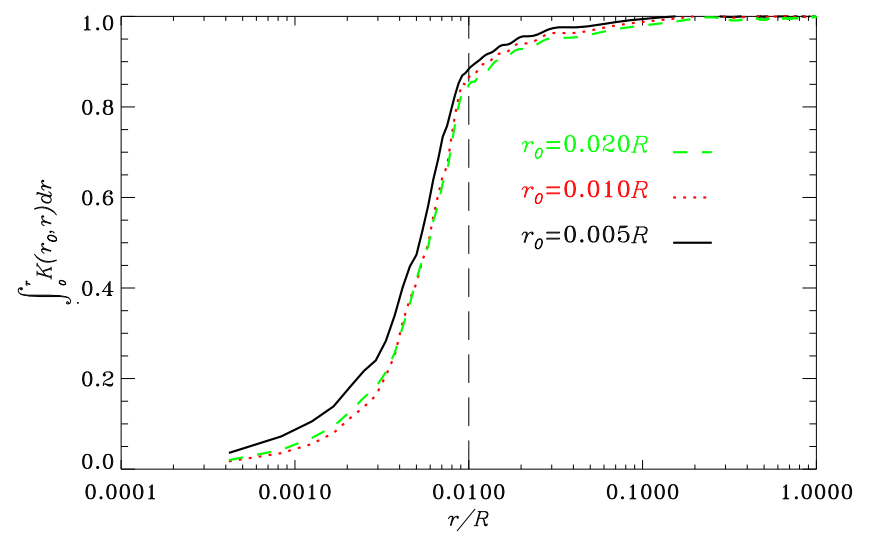

Figure 9. Cumulative integrals of the averaging kernels centered at different locations in the inner interior as obtained by the OLA inversion using Model 2. The dashed black line indicates the location of the inner edge of the H-burning shell.

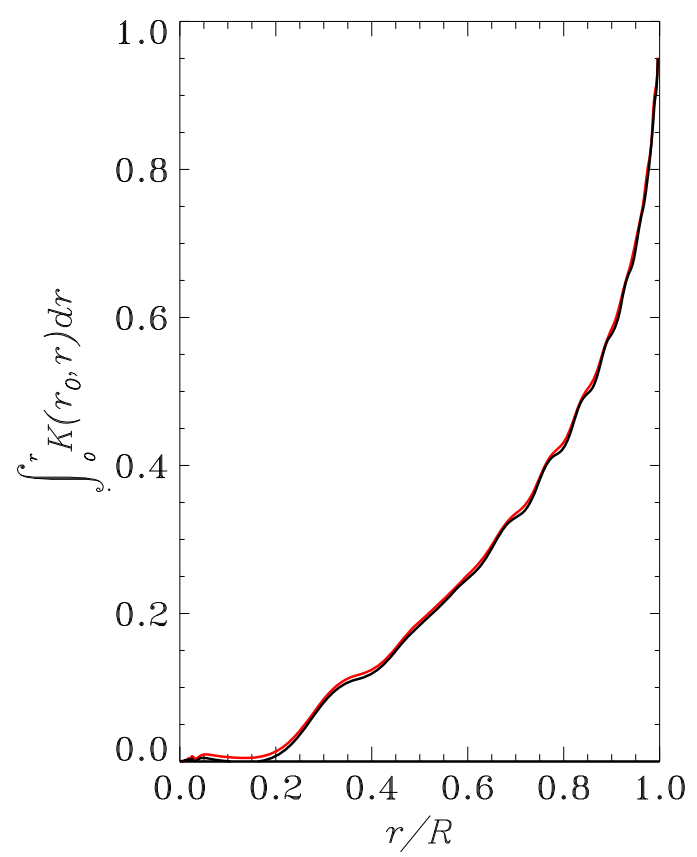

Figure 10. Cumulative integral of the surface averaging kernel (centered at $r=0.98 R$ ) obtained with the OLA method using Model 1 (red line) and Model 2 (black line).

Mauro et al. (2003) for the case of Procyon A. We computed the expected frequency splittings for several very simple rotational profiles by solving Equation (3) and adopting the kernels computed from the models used in the present work. Each set of data includes 14 artificial rotational splittings corresponding to the modes observed for KIC 4448777. A reasonable error of $7.8 \mathrm{nHz}$, equal for each rotational splitting, has been adopted (see Table 2). The sets of artificial splittings have then been inverted following the procedures as described in the above sections.

In our tests we used four different input rotational laws: (a) $\Omega$ $(r)=\Omega_{0}$; (b) $\Omega(r)=\Omega_{c}$ for $r \leqslant r_{c}, \Omega(r)=\Omega_{e}$ for $r>r_{c}$; (c) $\Omega(r)=\Omega_{0} \cos (2 \pi A r)$ where $A$ is a constant; (d) $\Omega(r)=$ $\Omega_{0}+\Omega_{1} r+\Omega_{2} r^{2}+\Omega_{3} r^{3}$.

Figure 15 shows the input rotational profiles and superimposed are the results obtained by OLA and SOLA inversions for four of the cases considered with the use of Model 2.

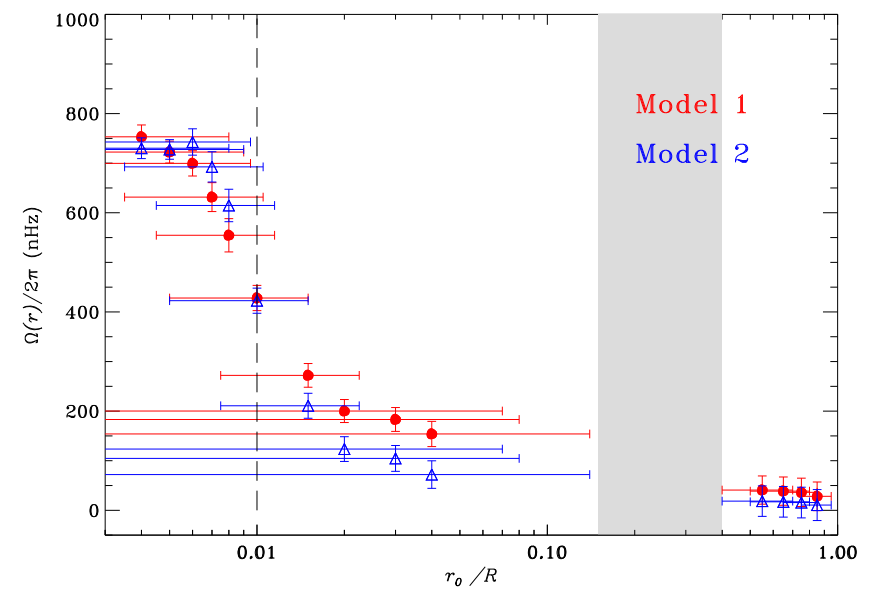

Figure 11. Internal rotation of KIC 4448777 at different target radii $r_{0}$ as obtained from the SOLA inversion for Models 1 and 2. The trade-off parameter used is $\mu=1$. Vertical error bars are two standard deviations. The dashed line indicates the location of the inner edge of the H-burning shell. The shaded area indicates the region inside the star in which it was not possible to determine any solutions.

Similar solutions have been obtained with Model 1. It should be pointed out that, although the panels show inversion results obtained along the entire profile to strengthen the potential of the inversion techniques, as already explained in Section 6, the considered set of dipolar modes allows one to probe properly only the regions where the modes are mostly localized.

We find that both the OLA and SOLA techniques are able to reproduce well the angular velocity in the core at $0.005 R$ and in the convective zone for $r \geqslant 0.4 R$, producing results that are in good agreement and model-independent. The OLA and SOLA techniques recover well rotational profiles that have convective envelopes or entire interiors that rotate rigidly. Figures 15(b), (c), and (d) show that both techniques are able to measure the maximum gradient strength of an internal rotational profile with a steep discontinuity, in the cases of both decreasing and increasing gradient toward the surface. However, the method fails to localize discontinuities with an accuracy better than $0.1 R$, due to the progressively increasing errors in spatial resolution at increasing distance from the core. In the case of the OLA technique the inverted rotational profile decreases smoothly toward the surface even in the case of a step-like input rotational law. On the other hand, with the SOLA inversion we find it more difficult to localize averaging kernels at target radii near the layer of strong gradient of angular velocity. See, for example, results plotted in Figures 15(b), (c), and (d), obtained for internal profiles characterized by discontinuities with different slopes.

In addition we notice that the inversion of the observed set of dipolar modes is not able to recover more complicated rotational profiles (such as the case of Figure 15(d)).

We can conclude from our tests that, with a small set of only dipolar modes, we have sufficient information to study the general properties of the internal rotational profile of a red giant, mainly the maximum gradient strength and, with some uncertainty, also the approximate radial location of the peak gradient. With the actual set of modes we are not able to distinguish between a smooth and a sharp rotation gradient inside our star. 

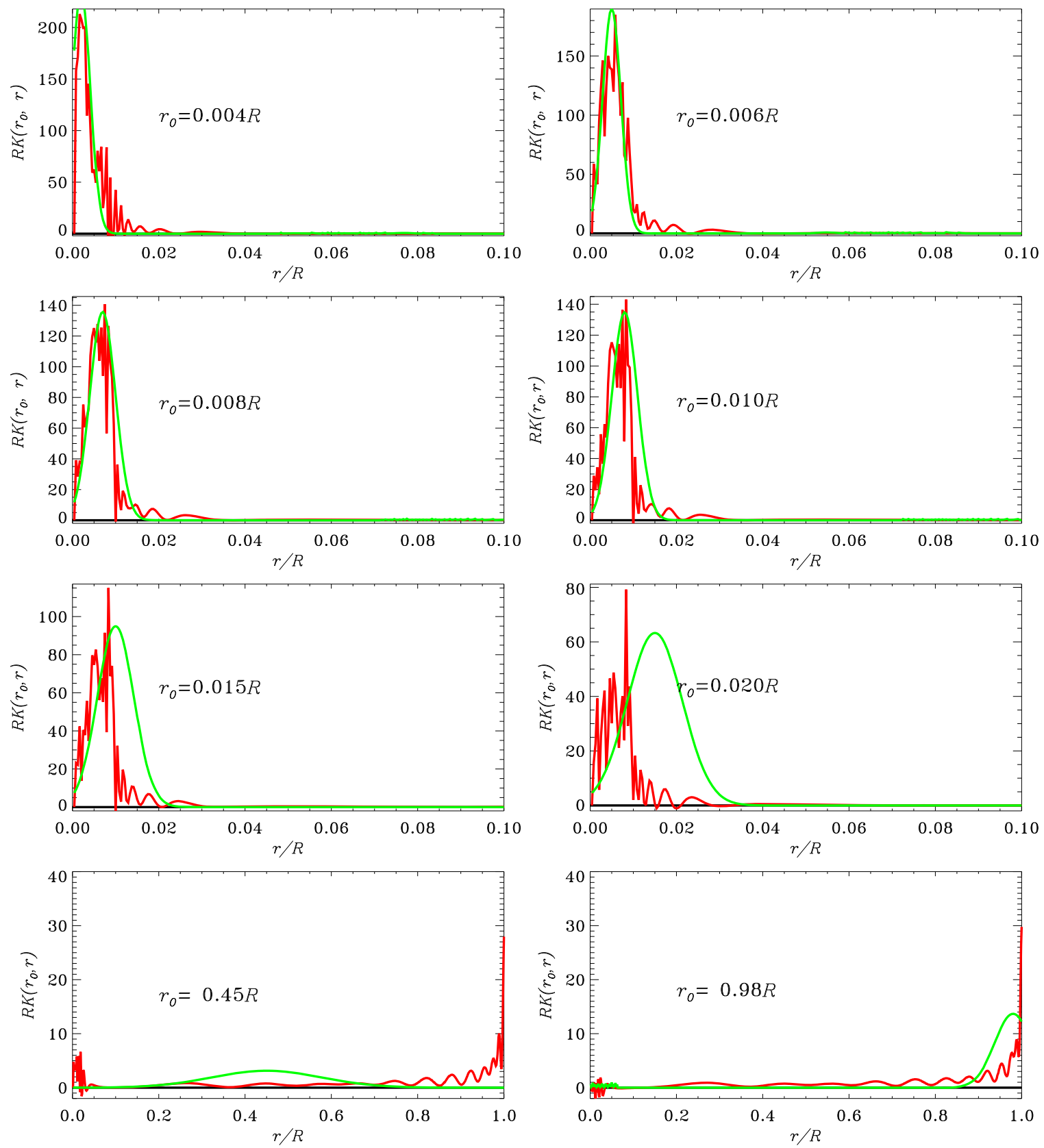

Figure 12. Averaging kernels (in red) plotted together with the Gaussian target functions (in green) for the SOLA inversion of KIC 4448777, adopting Model 2 and a trade-off parameter $\mu=1$.

\section{INTERNAL ROTATION BY OTHER METHODS}

As we have pointed out in the previous section, asteroseismic inversion of a set of 14 dipole-mode rotational splittings enables an estimate of the angular velocity only in the core and, to some degree, in some part of the radiative interior and in the convective envelope of the red-giant star.

Here we explore the possibility of comparing our inversion results and getting additional conclusions on the rotational velocity of the interior by applying different methods. This can be achieved by separating in our splittings data the contribution due to the rotation in the radiative region from that of the convective zone.

Recently, Goupil et al. (2013) proposed a procedure to investigate the internal rotation of red giants from the observations. They found that an indication of the average rotation in the envelope and the radiative interior can be obtained by estimating the trapping of the observed modes through the parameter $\zeta=I_{g} / I$, the ratio between the inertia in the gravity-mode cavity and the total inertia (see Equation (5)).

Because of the sharp decrease of the Brunt-Väisälä frequency at the edge of the H-burning shell, the gravity cavity corresponds to the radiative region, while the convective envelope essentially corresponds to the acoustic resonant cavity (see Figure 4). Thus, Goupil et al. (2013) demonstrated that, for $l=1$ modes, Equation (3) can be written as

$$
2 \pi \delta \nu_{n, l}=0.5 \zeta \Omega_{g}+(1-\zeta) \Omega_{p}=\zeta\left(0.5 \Omega_{g}-\Omega_{p}\right)+\Omega_{p},
$$



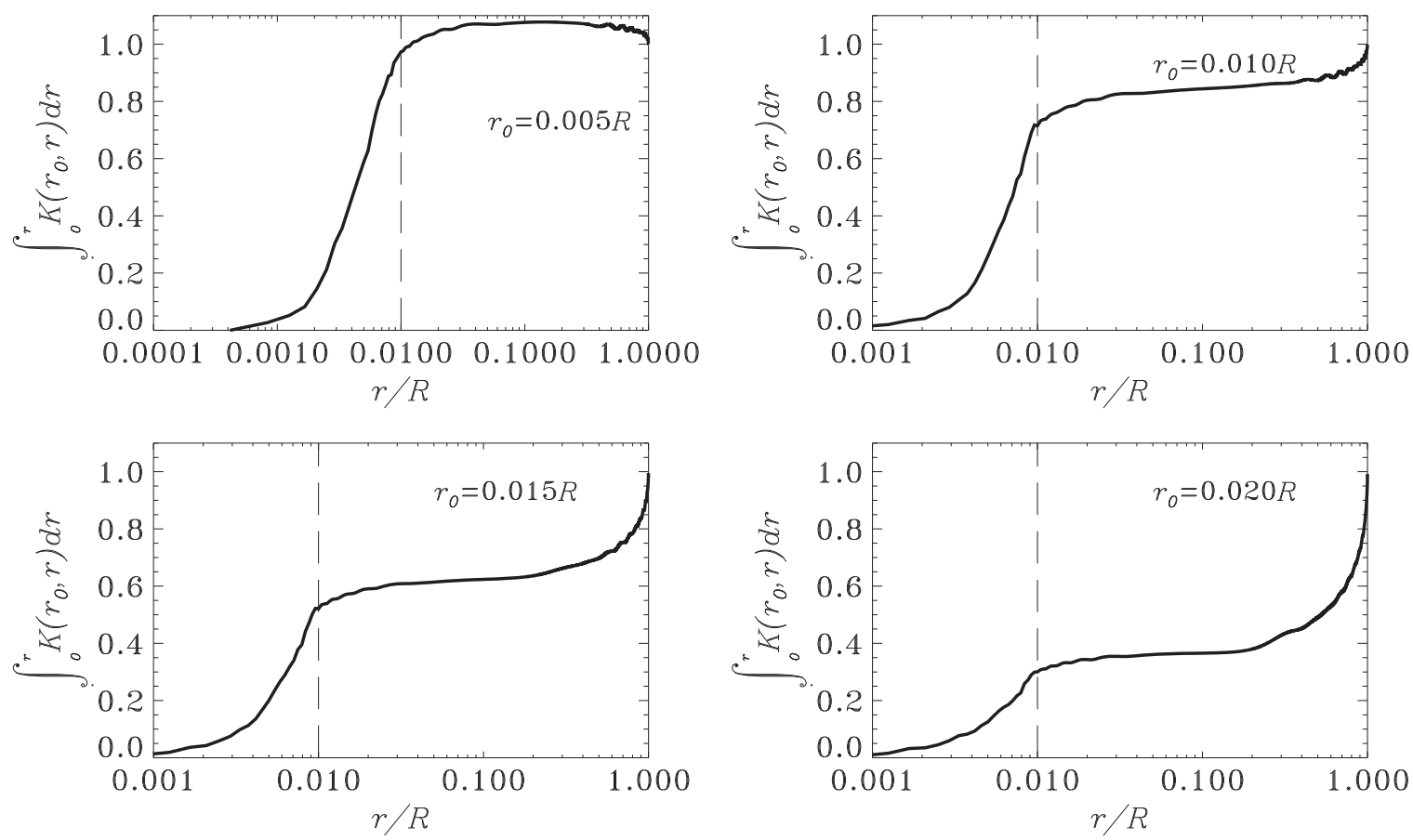

Figure 13. Cumulative integrals of the averaging kernels centered at different locations in the core obtained with the SOLA method using Model 2. The dashed line indicates the location of the inner edge of the H-burning shell.

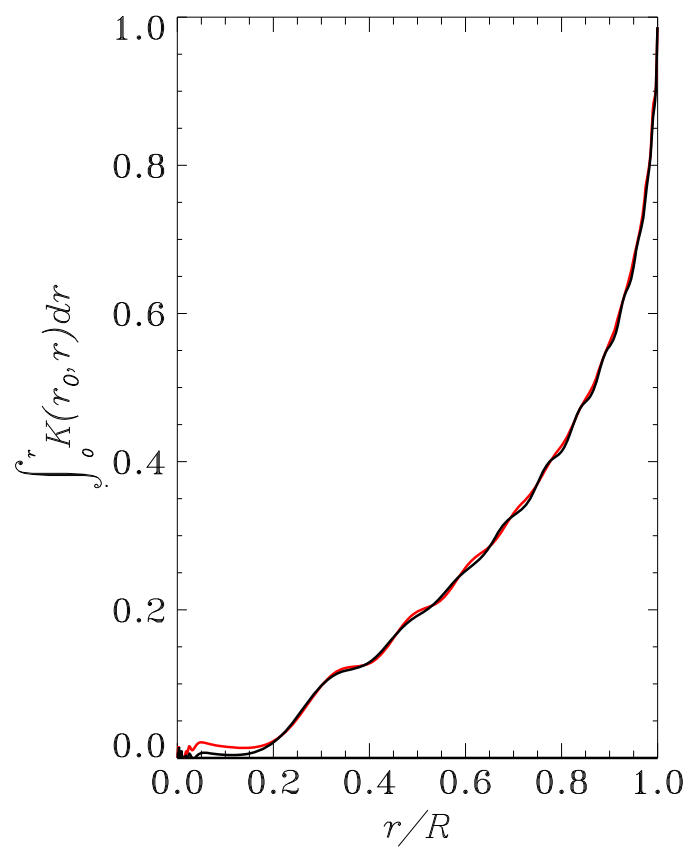

Figure 14. Cumulative integral of the surface averaging kernel (centered at $r_{0}=0.85 R$ ) obtained with the SOLA method using Model 1 (red line) and Model 2 (black line).

where $\Omega_{g}$ is the angular velocity averaged over the layers enclosed within the radius $r_{g}$ of the gravity cavity, while $\Omega_{p}$ is the mean rotation in the acoustic mode cavity. Equation (18) shows that an approximately linear relation exists between the observed rotational splittings and the trapping of the corresponding modes.

The parameter $\zeta$ has been computed for both Model 1 and Model 2 from the relevant eigenfunctions. In order to ascertain the model independence of the results we also computed $\zeta$ by adopting the approximated expression given by Goupil et al. (2013), based on the observed values of $\nu_{\max }, \Delta \nu$, and $\Delta \Pi_{1}$ (see Section 2). Figure 16 shows the linear dependence of the observed rotational splittings on $\zeta$ for both models and for Goupil's approximation.

We deduced the mean rotational velocity in the gravity cavity $(0<r<0.15 R), \Omega_{g} / 2 \pi$, as well as the mean rotational velocity in the acoustic cavity, $\Omega_{p}$, by fitting a relation of the type $\delta \nu=a \zeta+b$ to the observations, so that $\Omega_{g} / 2 \pi=2(a+b)$ and $\Omega_{p} / 2 \pi=b$. We obtained $\Omega_{g} / 2 \pi$ $=762.0 \pm 31.82 \mathrm{nHz}$ and $\Omega_{p} / 2 \pi=10.06 \pm 9.64 \mathrm{nHz}$ for Model 1 , and $\Omega_{g} / 2 \pi=756.48 \pm 29.86 \mathrm{nHz}$ and $\Omega_{p} / 2 \pi$ $=38.45 \pm 9.03 \mathrm{nHz}$ for Model 2. The results obtained by adopting Goupil's approximation for $\zeta$ are $\Omega_{g} / 2 \pi=748.90$ $\pm 36.30 \mathrm{nHz}$ and $\Omega_{p} / 2 \pi=-60.95 \pm 11.51 \mathrm{nHz}$.

It is clear that the determination of the mean rotation in the convective envelope of KIC 4448777 is very difficult by adopting this method, and we can only conclude that the value of $\Omega_{p}$ is certainly lower than the angular velocity of the core.

Another procedure to assess the angular velocity in the interior is by searching for the rotation profile that gives the closest match to the observed rotational splittings by performing a least-squares fitting. To do that, the stellar radius was cut into $K$ regions delimited by the radii $0=r_{0}<r_{1}<r_{2}<\ldots r_{K}=R$. Thus, Equation (3) can be modified in the following way:

$$
2 \pi \delta \nu_{i}=\int_{0}^{R} \mathcal{K}_{i}(r) \Omega(r) d r=\sum_{k=1}^{K} S_{i, k} \Omega_{k}
$$

where $S_{i, k}=\int_{k} \mathcal{K}_{i}(r) d r$ and $\mathcal{K}_{i}(r)$ are given by Equation (4) for each mode $i$ of the set of data used. $\Omega_{k}$ represents an average value of the angular velocity in the region $k$. 

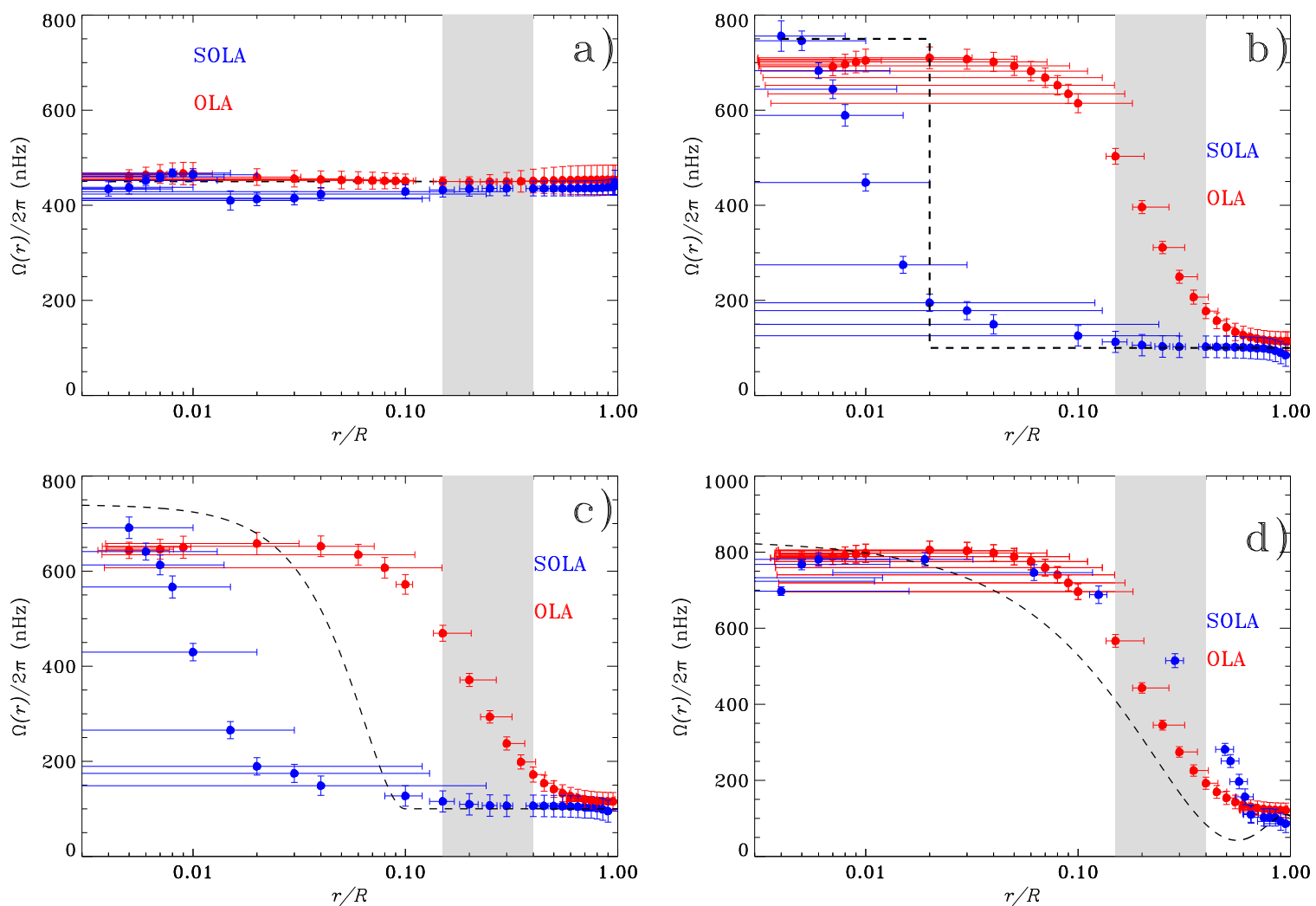

Figure 15. Internal rotation of Model 2 at different radii as obtained by the OLA and SOLA inversion of artificial rotational splittings calculated from four simple input rotational profiles shown by dashed lines. For solution above $r=0.1 R$ the horizontal error bars correspond to $10 \%$ of the radial spatial resolution defined by the averaging kernels. Profiles: (a) $\Omega(r) / 2 \pi=450 \mathrm{nHz} ; \quad$ (b) $\Omega(r) / 2 \pi=750 \mathrm{nHz} \quad$ for $r \leqslant 0.02 \quad$ and $\quad \Omega(r) / 2 \pi=100 \mathrm{nHz} \quad$ for $\quad r>0.02$; (c) $\Omega(r) / 2 \pi=740 \cos (2 \pi 0.5 r) \mathrm{nHz} ;$ (d) $\Omega(r) / 2 \pi=832-3515 r+4992 r^{2}-2202 r^{3} \mathrm{nHz}$. The shaded area indicates the region inside the star in which it was not possible to determine any solutions.

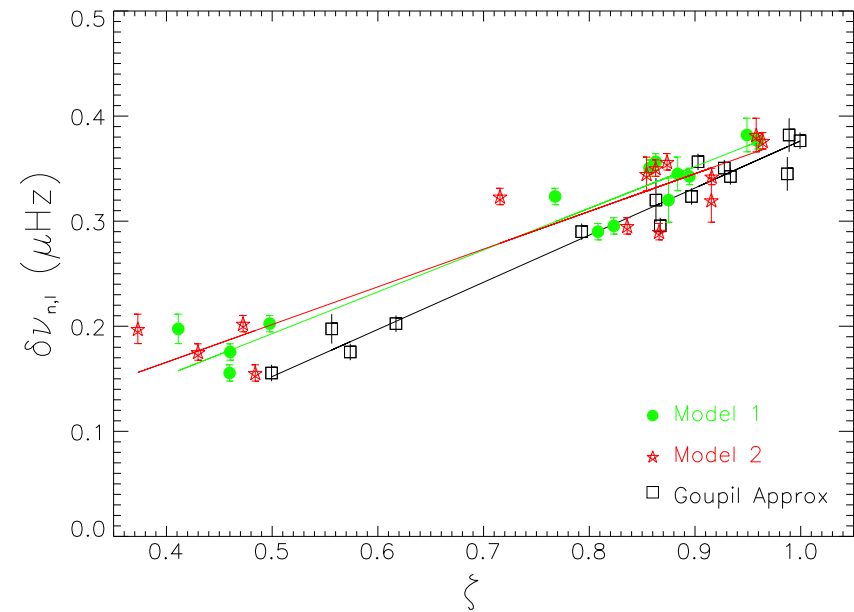

Figure 16. Observed splittings (Model 1: green bullets, Model 2: red stars, Goupil's approximation: black squares) plotted as a function of the parameter $\zeta$, which indicates the trapping of the modes. The solid lines shows a linear fit of the type $\delta \nu=a \zeta+b$.

To assess the angular velocity in the interior we can perform a least-squares fit to the observations by minimizing the $\chi^{2}$ function:

$$
\chi^{2}=\sum_{i=1}^{N} \frac{\left[2 \pi \delta \nu_{i}-\sum_{k=1}^{K} S_{i, k} \Omega_{k}\right]^{2}}{\epsilon_{i}^{2}} .
$$

We have explored several cases for small values of $K$ and we found that the result depends on $K$ and on the choice of the
Table 4

Averaged Rotation Rates Obtained by Least-square Fitting

\begin{tabular}{lcc}
\hline \hline & Model 1 & Model 2 \\
\hline$\Omega_{1} / 2 \pi(\mathrm{nHz})$ & $765 \pm 23$ & $746 \pm 22$ \\
$\Omega_{2} / 2 \pi(\mathrm{nHz})$ & $25 \pm 8$ & $52 \pm 8$ \\
\hline
\end{tabular}

values $r_{k}$ of the boundaries between each region $k$. Reasonable values of the reduced $\chi^{2}$ for the two models (Model 1: $\chi^{2}=5.10$, Model 2: $\chi^{2}=10.6$ ) have been obtained by dividing the interior of our models into two regions, so that the region for $k=1$ corresponds to radiative region $r / R \leqslant 0.15$, while the region for $k=2$ corresponds to the convective envelope with $0.15<r / R \leqslant 1.0$.

Table 4 lists the values of $\Omega_{k}$ in the different regions for the two models. Once the least-squares problem has been solved, we can use the coefficients to calculate the averaging kernels:

$$
K\left(r_{k}, r\right)=\sum_{i=1}^{N} c_{i}\left(r_{k}\right) \mathcal{K}_{i}(r)
$$

Figure 17 shows that while the cumulative integrated kernel of the region $k=1$ is quite sensitive to the core, although a contribution from the surface is still present, the cumulative integrated kernel of the region $k=2$ is strongly contaminated by the modes trapped in the radiative interior. 


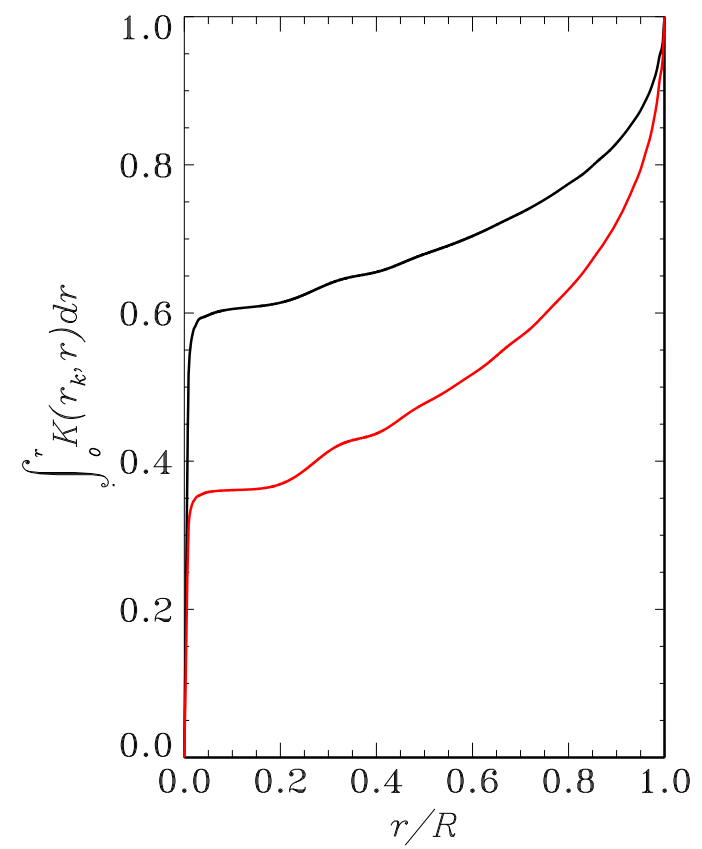

Figure 17. Cumulative integral of the averaging kernel of the core (black line) and the surface (red line) obtained with the LS method using Model 2.

\section{CONCLUSION}

In the present paper, we have analyzed the case of KIC 4448777, a star at the beginning of the red-giant phase, for which a set of only 14 rotational splittings of dipolar modes have been identified. Its internal rotation has been probed successfully by means of asteroseismic inversion.

We confirm previous findings obtained in other red giants (Beck et al. 2012; Deheuvels et al. 2012, 2014) that the inversion of rotational splittings can be employed to probe the angular velocity not only in the core but also in the convective envelope. We find that the helium core of KIC 4448777 rotates faster than the surface at an angular velocity of about $\left\langle\Omega_{c} / 2 \pi\right\rangle=748 \pm 18 \mathrm{nHz}$, obtained as an average value from the SOLA and OLA inversion techniques. Moreover, we found that the result in the core, well probed by mixed modes, does not depend on the model of stellar structure employed in the inversion. The value estimated in the core agrees well with those obtained by applying other methods, such as the one based on the relation between the observed rotational splittings and the inertia of the modes (Goupil et al. 2013), or the leastsquares fit to the observed rotational splittings.

Our results have shown that the mean rotation in the convective envelope of KIC 4448777 is $\left\langle\Omega_{s} / 2 \pi\right\rangle=68 \pm 22 \mathrm{nHz}$, obtained as an average of the OLA inversion results for the two "best-fitting" selected models, indicating that the core should rotate about 11 times faster than the convective envelope. For the SOLA inversion solutions, it was not possible to suppress efficiently the strong signal of the modes concentrated in the core. The value of the rotation deduced in the convective zone is compatible with the upper limit measured at the photosphere $\Omega_{\mathrm{ph}} / 2 \pi<538 \mathrm{nHz}$ derived from the spectroscopic value of $v$ sin $i$ (see Section 2) and the stellar radius provided by the models. Unfortunately, with few modes able to probe the acoustic cavity efficiently, we have been able to deduce only a weighted average of the rotation in the whole convection zone, but the inversion results appear not to depend on the equilibrium structure model employed. Other methods used to determine rotation in the convection zone have provided us with results in reasonable agreement with those obtained by inversion.

Furthermore, we demonstrate that the inversion of rotational splittings can be employed to probe the variation with radius of the angular velocity in the core, because the observed mixed modes enable us to build well-localized averaging kernels. The application of both SOLA and OLA inversion techniques allowed us to show that the entire core is rotating with a constant angular velocity. In addition, the SOLA method found evidence for a decrease in angular velocity occurring in the region $0.007 R \leqslant r \leqslant 0.1 R$, between the helium core and part of the hydrogen-burning shell, which cannot be better localized, due to the intrinsic limits of the applied technique and to the lower resolving power of the employed modes in the regions above the core. Thus, although we are not able to distinguish between a smooth and a sharp gradient, we can determine to a good approximation the maximum gradient strength and the radial position of the peak gradient.

With the available data, including just a modest number of dipolar modes, it is clearly impossible to infer the complete internal rotation law of KIC 4448777. In order to resolve the regions above the core, it is necessary to invert a set of data that includes more rotational splittings, and in particular of modes with $l>1$ and with significant amplitudes in the acoustic cavity. Such data may become available for other targets or from analysis of longer time-series observations than those considered here.

It seems fair to say that, at this stage, the asteroseismic inversions are giving very useful results to test the actual evolution theory of stellar structure, but we expect that the ever-improving accuracy of the data will drive the theory to advance in new directions and eventually lead to a more thorough understanding of stellar rotation. Considering the fact that the internal angular velocity of the core of the red giants is theoretically expected to be higher than our results, it remains necessary to investigate more efficient mechanisms of angular momentum transport acting on the appropriate timescales during the different phases of the stellar evolution, before the red-giant phase. We expect that measurements of rotational splittings for modes with low inertia will shed some light on the above picture and on the question of the stellar angular momentum transport. Although some preliminary tests (Beck et al. 2014) have shown that the use of $l=2$ mode splittings cannot help to resolve internal rotation inside red giants, we believe that a detailed analysis is required by considering red giants at different evolutionary phases.

We conclude that it is reasonable to think that this approach, proved to be very powerful in the case of the Sun, for which thousands of modes from low to high degree have been detected, can be applied well even to small sets of only dipolar modes in red-giant stars.

The authors thank the anonymous referee very much for his/ her suggestion and comments, which gave the opportunity to greatly improve the manuscript.

Funding for the Kepler mission is provided by NASA's Science Mission Directorate. We thank the entire Kepler team for the development and operations of this outstanding mission.

W.A.D. was supported by the Polish NCN grant DEC-2012/ 05/B/ST9/03932. S.H. acknowledges financial support from 
the Netherlands Scientific Organization (NWO). D.S. acknowledges support from the Australian Research Council. Funding for the Stellar Astrophysics Centre is provided by The Danish National Research Foundation (Grant agreement no.: DNRF106). The research is supported by the ASTERISK project (ASTERoseismic Investigations with SONG and Kepler) funded by the European Research Council (Grant agreement no.: 267864). B.M., P.B., and R.A.G. acknowledge the ANR (Agence Nationale de la Recherche, France) program IDEE (n. ANR-12-BS05-0008) "Interaction Des Étoiles et des Exoplanètes." A.T. is postdoctoral fellow of the Fund for Scientific Research (FWO), Flanders, Belgium. The research leading to the presented results has received funding from the European Research Council under the European Community's Seventh Framework Programme (FP7/2007-2013)/ERC grant agreement no 338251 (StellarAges). The work presented here is also based on ground-based spectroscopic observations made with the Mercator Telescope, operated on the island of $\mathrm{La}$ Palma by the Flemish Community, at the Spanish Observatorio del Roque de los Muchachos of the Instituto de Astrofisica de Canarias.

M.P.D.M. is grateful to the Astrophysical Observatory of Catania for hosting her during the preparation of the present manuscript.

\section{REFERENCES}

Angulo, C., Arnould, M., Rayet, M., et al. 1999, NuPhA, 656, 3

Backus, G. E., \& Gilbert, F. 1970, RSPTA, 266, 123

Baglin, A., Auvergne, M., Boisnard, L., et al. 2006, in 36th COSPAR Scientific Assembly Plenary Meeting 36, Meeting abstract from the CDROM (Beijing: ESA), 3749

Beck, P. G. 2013, PhD thesis, Arenberg Doctoral School of Science, Engineering \& Technology, Faculty of Science, Department of Physics and Astronomy, KU Leuven, Belgium

Beck, P. G., Bedding, T. R., Mosser, B., et al. 2011, Sci, 332, 205

Beck, P. G., Hambleton, K., Vos, J., et al. 2014, A\&A, 564, 36

Beck, P. G., Montalban, J., Kallinger, T., et al. 2012, Natur, 481, 55

Bedding, T. R., Mosser, B., Huber, D., et al. 2011, Natur, 471, 608

Belkacem, K., Goupil, M. J., Dupret, M. A., et al. 2011, A\&A, 530, 142

Berthomieu, G., Toutain, T., Gonczi, G., et al. 2001, in ESA SP-464, Proc. SOHO 10/GONG 2000 Workshop Helio- and Asteroseismology at the Dawn of the Millennium, ed. A. Wilson (Noordwijk: ESA Publications Division), 411

Böhm-Vitense, E. 1958, ZA, 46, 108

Borucki, W. J., Koch, D., Basri, G., et al. 2010, Sci, 327, 977

Brandão, I. M., Dog`an, G., Christensen-Dalsgaard, J., et al. 2011, A\&A, 527, A37

Brun, A. S., \& Zahn, J. P. 2006, A\&A, 457, 665

Cantiello, M., Mankovich, C., Bildsten, L., et al. 2014, ApJ, 788, 93

Castelli, F., \& Kurucz, R. L. 2004, A\&A, 419, 725

Ceillier, T., Eggenberger, P., García, R. A., \& Mathis, S. 2013, A\&A, 555, 54

Chaboyer, B., Demarque, P., \& Pinsonneauls, M. H. 1995, ApJ, 441, 876

Chaplin, W. J., Eslworth, Y., Isaak, G. R., et al. 2002, MNRAS, 336, 979

Christensen-Dalsgaard, J. 2008a, Ap\&SS, 316, 13

Christensen-Dalsgaard, J. 2008b, Ap\&SS, 316, 113

Christensen-Dalsgaard, J., Schou, J., \& Thompson, M. 1990, MNRAS, 242, 353

Corsaro, E., \& De Ridder, J. 2014, A\&A, 571, 71

Corsaro, E., et al. 2012, ApJ, 757, 190

Cowling, T. G., \& Newing, R. A. 1949, ApJ, 109, 149

Deheuvels, S., Dogan, G., Goupil, M. J., et al. 2014, A\&A, 564, 27
Deheuvels, S., García, R. A., Chaplin, W. J., et al. 2012, ApJ, 756, 19 De Smedt, K., Van Winckel, H., Karakas, A. I., et al. 2012, A\&A, 541, 67

Di Mauro, M. P. 2004, in ESA-SP 559, Proc. SOHO 14/GONG 2004 Workshop Helio- and Asteroseismology: Towards a Golden Future, ed. D. Danesy, 186

Di Mauro, M. P., Cardini, D., Catanzaro, G., et al. 2011, MNRAS, 415, 3783

Di Mauro, M. P., Christensen-Dalsagaard, J., \& Weiss, A. 2003, in Proc. Workshop, The Third MONS Workshop: Science Preparation and Target Selection, ed. T. C. Teixeira \& T. R. Bedding (Aarhus: Aarhus Universitet), 151

Di Mauro, M. P., \& Dziembowski, W. 1998, MmSAI, 69, 559

Elsworth, Y., Howe, R., Isaak, G. R., et al. 1995, Natur, 376, 669

García, R. A., Ceillier, T., Salabert, D., et al. 2014, A\&A, 572, 34

García, R. A., Hekker, S., Stello, D., et al. 2011, MNRAS, 414, L6

Gough, D. O. 1981, MNRAS, 196, 731

Gough, D. O., \& Kosovichev, A. G. 1993, in ASP Conf. Ser. 40, IAU Coll. 137, "Inside the Stars", ed. T. M. Brown (San Francisco, CA: ASP), 541 Goupil, M. J. 2009, LNP, 765, 45

Goupil, M. J., Dziembowski, W. A., Goode, P. R., et al. 1996, A\&A, 305, 487

Goupil, M. J., Mosser, B., Marques, J. P., et al. 2013, A\&A, 549, A75

Grevesse, N., \& Noels, A. 1993, in Symposium in Honour of Hubert Reeves' 60th Birthday, Origin and Evolution of the Elements, ed. S. Kubono \& T. Kajino (Cambridge: Cambridge Univ. Press), 14

Handberg, R., \& Campante, T. L. 2011, A\&A, 527, A56

Huber, D., Ireland, M. J., Bedding, T. R., et al. 2012, ApJ, 760, 32

Huber, D., Stello, D., Bedding, T. R., et al. 2009, CoAst, 160, 74

Iglesias, C. A., \& Rogers, F. J. 1996, ApJ, 464, 943

Jenkins, J. M., Caldwell, D. A., Chandrasekaran, H., et al. 2010, ApJL, 713, L87

Kallinger, T., Mosser, B., Hekker, S., et al. 2010, A\&A, 522, A1

Kawaler, S. D., Sekii, T., \& Gough, D. 1999, ApJ, 516, 349

Kjeldsen, H., \& Bedding, T. 1995, A\&A, 293, 87

Kjeldsen, H., Bedding, T. R., \& Christensen-Dalsgaard, J. 2008, ApJL, 683, L175

Kupka, F. G., Ryabchikova, T. A., Piskunov, N. E., et al. 2000, BaltA, 9, 590 Lochard, J., Samadi, R., \& Goupil, M. J. 2005, A\&A, 438, 939

Marques, J. P., Goupil, M. J., Lebreton, Y., et al. 2013, A\&A, 549, A74

Mathur, S., Bruntt, H., Catala, C., et al. 2013, A\&A, 549, 12

Mathur, S., Hekker, S., Trampedach, R., et al. 2011, ApJ, 741, 119

Miglio, A., Brogaard, K., Stello, D., et al. 2012, MNRAS, 419, 2077

Mosser, B., Barban, C., Montalban, J., et al. 2011, A\&A, 532, A86

Mosser, B., Dziembowski, W. A., Belkacem, K., et al. 2013a, A\&A, 559, 137

Mosser, B., Goupil, M. J., Belkacem, K., et al. 2012a, A\&A, 540, A143

Mosser, B., Goupil, M. J., Belkacem, K., et al. 2012b, A\&A, 548, A10

Mosser, B., Michel, E., Belkacem, K., et al. 2013b, A\&A, 550, 126

Paternò, L., Sofia, S., \& Di Mauro, M. P. 1996, A\&A, 314, 940

Phillips, D. L. 1962, JCoPh, 1, 84

Pijpers, F. P., \& Thompson, M. J. 1992, A\&A, 262, L33

Raskin, G., van Winckel, H., Hensberge, H., et al. 2011, A\&A, 526, 69

Rogers, F. J., \& Nayvonov, A. 2002, ApJ, 576, 1064

Roxburgh, I. W., Audard, N., Basu, S., et al. 1998, in IAU Symp. 181, Sounding Solar and Stellar Interiors (poster vol.) Nice Observatory, ed. J. Provost, \& F.-X. Schmider (Nice: Nice Observatory), 245

Schou, J., Antia, H. M., Basu, S., et al. 1998, ApJ, 505, 390

Sekii, T. 1997, in IAU Symp. No. 181, Sounding Solar and Stellar Interiors, ed. J. Provost \& F.-X. Schmider (Dordrecht: Kluwer), 189

Stello, D. 2012, in ASP Conf. Proc. 462, Progress in Solar/Stellar Physics with Helio- and Asteroseismology, ed. H. Shibahashi, M. Takata, \& A. E. Lynus-Gray (San Francisco, CA: ASP), 200

Stello, D., Huber, D., Bedding, T. R., et al. 2013, ApJL, 765, L41

Suárez, J. C., Andrade, L., Goupil, M. J., et al. 2010, AN, 331, 1073

Talon, S., \& Charbonnel, C. 2005, A\&A, 440, 981

Tayar, J., \& Pinsonneault, M. H. 2013, ApJL, 775, 1

Thompson, M. J., Christensen-Dalsgaard, J., Miesch, M. S., \& Toomre, J. 2003, ARA\&A, 41, 599

Thompson, M. J., Toomre, J., Anderson, E. R., et al. 1996, Sci, 272, 1300

Tikhonov, A. N. 1963, Sov. Maths., 4, 1035 